\title{
A Wide and Deep Exploration of Radio Galaxies with Subaru HSC (WERGS). IV. Rapidly Growing (Super)Massive Black Holes in Extremely Radio-loud Galaxies
}

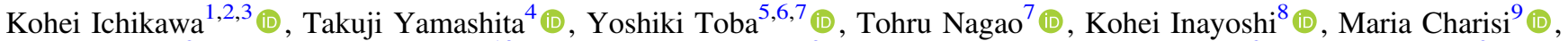

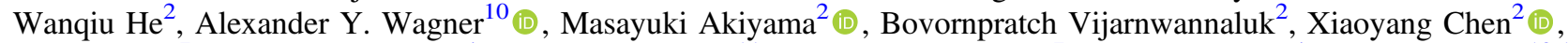 \\ Masaru Kajisawa ${ }^{7}$, Taiki Kawamuro ${ }^{4}$ (), Chien-Hsiu Lee ${ }^{11}$ (10), Yoshiki Matsuoka ${ }^{7}$, Malte Schramm ${ }^{4}$ (1) , Hyewon Suh ${ }^{12,13}$ (1) \\ Masayuki Tanaka ${ }^{4}$ (D) Hisakazu Uchiyama ${ }^{4}$, Yoshihiro Ueda ${ }^{5}$ (D) Janek Pflugradt ${ }^{2}$, and Hikaru Fukuchi ${ }^{2}$ \\ ${ }^{1}$ Frontier Research Institute for Interdisciplinary Sciences, Tohoku University, Sendai 980-8578, Japan; k.ichikawa@ astr.tohoku.ac.jp \\ 2 Astronomical Institute, Tohoku University, Aramaki, Aoba-ku, Sendai, Miyagi 980-8578, Japan \\ ${ }^{3}$ Max-Planck-Institut für extraterrestrische Physik (MPE), Giessenbachstrasse 1, D-85748 Garching bei Müunchen, Germany \\ ${ }^{4}$ National Astronomical Observatory of Japan, 2-21-1 Osawa, Mitaka, Tokyo 181-8588, Japan \\ ${ }^{5}$ Department of Astronomy, Kyoto University, Kitashirakawa-Oiwake-cho, Sakyo-ku, Kyoto 606-8502, Japan \\ ${ }^{6}$ Academia Sinica Institute of Astronomy and Astrophysics, $11 \mathrm{~F}$ of Astronomy-Mathematics Building, AS/NTU, No.1, Section 4, Roosevelt Road, \\ Taipei 10617, Taiwan \\ ${ }^{7}$ Research Center for Space and Cosmic Evolution, Ehime University, 2-5 Bunkyo-cho, Matsuyama, Ehime 790-8577, Japan \\ ${ }^{8}$ Kavli Institute for Astronomy and Astrophysics, Peking University, Beijing 100871, People's Republic of China \\ ${ }^{9}$ TAPIR, California Institute of Technology, 1200 E. California Blvd., Pasadena, CA 91125, USA \\ ${ }^{10}$ Center for Computational Sciences, University of Tsukuba, 1-1-1 Tennodai, Tsukuba, Ibaraki 305-8577, Japan \\ ${ }^{11}$ NSF's National Optical-Infrared Astronomy Research Laboratory, Tucson, AZ 85719, USA \\ ${ }^{12}$ Subaru Telescope, National Astronomical Observatory of Japan (NAOJ), 650 North A'ohoku Place, Hilo, HI 96720, USA \\ ${ }^{13}$ Gemini Observatory/NSF's NOIRLab, 670 N. A'ohoku Place, Hilo, HI 96720, USA \\ Received 2020 May 3; revised 2021 July 19; accepted 2021 August 4; published 2021 October 29
}

\begin{abstract}
We present the optical and infrared properties of 39 extremely radio-loud galaxies discovered by cross-matching the Subaru/Hyper Suprime-Cam (HSC) deep optical imaging survey and VLA/FIRST 1.4 GHz radio survey. The recent Subaru/HSC strategic survey revealed optically faint radio galaxies $(\mathrm{RG})$ down to $g_{\mathrm{AB}} \sim 26$, opening a new parameter space of extremely radio-loud galaxies (ERGs) with radio-loudness parameter of $\log \mathcal{R}_{\text {rest }}=$ $\log \left(f_{1.4 \mathrm{GHz}, \text { rest }} / f_{g, \text { rest }}\right)>4$. Because of their optical faintness and small number density of $\sim 1 \mathrm{deg}^{-2}$, such ERGs were difficult to find in the previous wide but shallow or deep but small area optical surveys. ERGs show intriguing properties that are different from the conventional RGs: (1) most ERGs reside above or on the starforming main-sequence and some of them might be low-mass galaxies with $\log \left(M_{\star} / M_{\odot}\right)<10$. (2) ERGs exhibit a high specific black hole accretion rate, reaching the order of the Eddington limit. The intrinsic radio loudness $\left(\mathcal{R}_{\text {int }}\right)$, defined by the ratio of jet power over bolometric radiation luminosity, is one order of magnitude higher than that of radio quasars. This suggests that ERGs harbor a unique type of active galactic nuclei (AGN) that show both powerful radiations and jets. Therefore, ERGs are prominent candidates of very rapidly growing black holes reaching Eddington-limited accretion just before the onset of intensive AGN feedback.
\end{abstract}

Unified Astronomy Thesaurus concepts: Supermassive black holes (1663); Active galactic nuclei (16); Radio active galactic nuclei (2134)

\section{Introduction}

The formation of supermassive black holes (SMBHs) and their growth across the cosmic time are fundamental questions in modern astronomy. In the local universe at $z \sim 0$, the mass of $\mathrm{SMBH}\left(M_{\mathrm{BH}}\right)$ and their host properties show tight correlations for the SMBH mass range of $6 \lesssim \log \left(M_{\mathrm{BH}} / M_{\odot}\right) \lesssim 10$ with a scatter of $\sigma \sim 0.3$ dex (e.g., Magorrian et al. 1998; Ferrarese \& Merritt 2000; Gebhardt et al. 2000; Tremaine et al. 2002; Marconi \& Hunt 2003; Häring \& Rix 2004; Sani et al. 2011; Kormendy \& Ho 2013; McConnell \& Ma 2013). Such a tight correlation is considered to be established by a balance of feeding and feedback processes between the central SMBHs and the host galaxies.

Local radio galaxies have been primary targets for investigating the effect of SMBHs on the host galaxies because powerful radio galaxies or radio-loud active galactic nuclei (AGNs) mainly reside in massive galaxies whose star formation is quenched, with the presence of strong jets dispersing the interstellar medium (e.g., Morganti et al. 2005; Holt et al. 2008; Nesvadba et al. 2017) or producing cavities in the host galaxies (e.g., Rafferty et al. 2006;
McNamara \& Nulsen 2007; Bîrzan et al. 2008; Blandford et al. 2019). Those radio galaxies tend to show a low accretion rate, i.e., Eddington ratio of $\lambda_{\text {Edd }}<10^{-2}$, suggesting that the energy release is dominated by the kinetic power by jet, not by the radiation from the AGN accretion disk.

However, the situation may be different at $z>1$. Using over $10^{3}$ radio AGNs selected from the Very Large Array (VLA)COSMOS $3 \mathrm{GHz}$ large project (Smolčić et al. 2017a), Delvecchio et al. (2018) demonstrated that SMBH accretion in radio-bright $\left(L_{1.4 \mathrm{GHz}}>10^{25} \mathrm{WHz}^{-1}\right)$ AGNs becomes more radiatively efficient $\left(\lambda_{\mathrm{Edd}}>10^{-2}\right)$ at $z>1$. They reside in starforming galaxies, which contain plenty of cold gas. This picture of radio AGNs is completely different from those seen in the local universe in the same radio luminosity range (e.g., Hickox et al. 2009). Still, the survey volume of VLA-COSMOS surveys is small so they may be missing a rare, but radio-bright population. The FIRST survey is the best tool for exploring such a radio-bright end since it covers half the sky with the VLA at 1.4 GHz (e.g., Becker et al. 1995; Helfand et al. 2015). However, cross matching the VLA/FIRST sources with the SDSS survey 


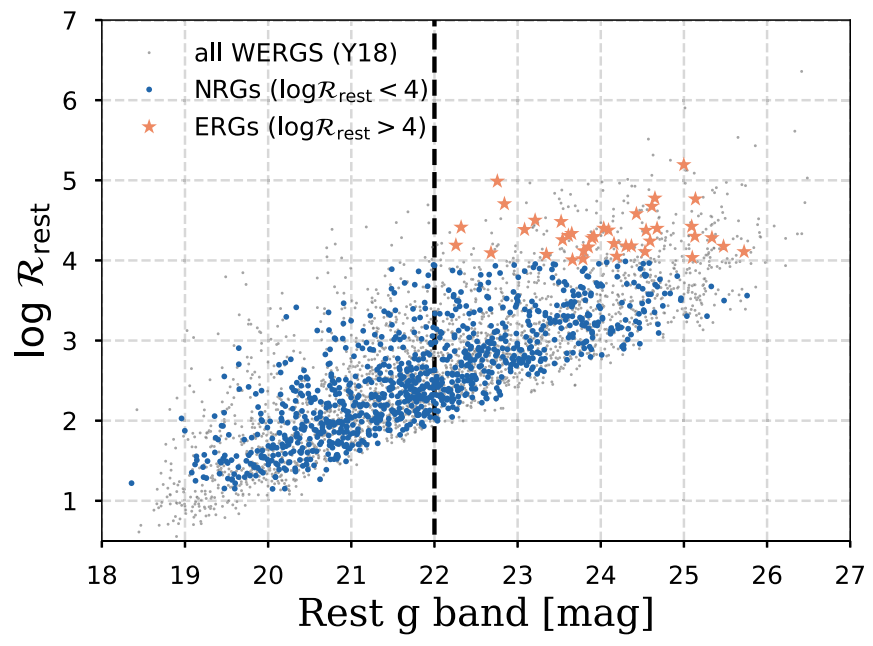

Figure 1. $\log \mathcal{R}_{\text {rest }}$ vs. rest $g$-band magnitude of our sample. The original WERGS sample obtained by Yamashita et al. (2018) is shown with gray circles. The finally selected 987 WERGS sample with $\log \mathcal{R}_{\text {rest }}<4$ used in this study (we call the sample "NRGs" in the text) and 39 ERGs (the sample with $\log \mathcal{R}_{\text {rest }}>4$ ) are shown with blue circles, and orange stars, respectively. The vertical dashed line indicates the magnitude limit of the SDSS survey at $g_{\mathrm{AB}}=22$.

catalog identified optical counterparts in only $30 \%$ of the radio sources (Ivezić et al. 2002; Best \& Heckman 2012).

A recent Subaru/Hyper Suprime-Cam (HSC; Miyazaki et al. 2018) strategic survey shed light on such a situation. We have conducted a search for optically faint radio galaxies (RGs) using the Subaru HSC survey catalog (Aihara et al. 2018a) and the VLA/FIRST radio continuum catalog, and we have found a large number $\left(>3 \times 10^{3}\right.$ sources $)$ of RGs at $z \sim 0-5$ (Yamashita et al. 2018, 2020). The project is called the Wide and deep Exploration of Radio Galaxies with Subaru/HSC (WERGS; Yamashita et al. 2018). Yamashita et al. (2018) demonstrated that over $60 \%$ of populations now have reliable optical counterparts thanks to deep HSC/optical imaging. Figure 1 shows that the WERGS sample spans a wide range of optical magnitude $g_{\mathrm{AB}}=18-26$ and radio-loudness parameter of $\log \mathcal{R}_{\text {rest }}=\log \left(f_{1.4 \mathrm{GHz}, \text { rest }} / f_{g \text { band,rest }}\right)=1-6 \quad$ (e.g., Ivezić et al. 2002).

Our particular interest is the new parameter space of optically faint RGs that are extremely radio-loud galaxies (ERGs) with $\log \mathcal{R}_{\text {rest }}>4$. Previously known radio quasars have a peak of radio loudness at $\log \mathcal{R}_{\text {rest }}=2-3$, and rarely contain any sources with $\log \mathcal{R}_{\text {rest }}>4$ (Ivezić et al. 2002; Inoue et al. 2017). Therefore, the optical emission of ERGs does not seem to originate from the AGN accretion disk anymore probably due to dust obscuration of the nuclei, but it traces the stellar component of the host galaxies (e.g., Terashima \& Wilson 2003). Given that the optical band is very faint with median magnitudes of $\left\langle g_{\mathrm{AB}}\right\rangle \sim 24.5$, ERGs might have smaller stellar masses than previously known radio sources. Considering the very small number density of ERGs $\left(\sim 1\right.$ source deg $\left.{ }^{-2}\right)$, it is not surprising that deep but smallervolume surveys (such as VLA-COSMOS Smolčić et al. 2017a, 2017b) have rarely detected such ERGs and the very wide SDSS survey (e.g., SDSS-selected radio galaxies, Best \& Heckman 2012) could not detect ERGs either because of their shallow sensitivity down to only $g_{\mathrm{AB}} \sim 22$.

In this study, we will explore the physical properties of ERGs. Toba et al. (2019) already compiled multi-wavelength
Table 1

Summary of the Sample Selection Cut in This Study

\begin{tabular}{|c|c|c|}
\hline $\begin{array}{l}\text { (1) } \\
\text { Selection }\end{array}$ & $\begin{array}{c}\text { (2) } \\
\text { No. of WERGS } \\
\text { Sources }\end{array}$ & $\begin{array}{c}\text { (3) } \\
\text { No. } \\
\text { of ERGs }\end{array}$ \\
\hline $\begin{array}{l}\text { Parent WERGS sample of Yamashita } \\
\text { et al. (2018) }\end{array}$ & 3579 & 273 \\
\hline$L_{1.4 \mathrm{GHz}}>10^{24} \mathrm{~W} \mathrm{~Hz}^{-1}$ & 3147 & 270 \\
\hline compact radio sources & 2792 & 208 \\
\hline $\begin{array}{l}\text { No nearby multiple FIRST sources } \\
\text { in }<1^{\prime}\end{array}$ & 2583 & 192 \\
\hline $\begin{array}{l}\text { No nearby multiple HSC sources } \\
\text { in }<1^{\prime \prime}\end{array}$ & 2573 & 187 \\
\hline Redshift range at $0.3<z<1.6$ & 2286 & 145 \\
\hline Good photo- $z$ quality & 1770 & 79 \\
\hline $\begin{array}{l}\text { IR available region by Toba et al. } \\
\text { (2019) }\end{array}$ & 1055 & 39 \\
\hline$q_{\mathrm{IR}}<1.68$ (final sample) & 1026 & 39 \\
\hline
\end{tabular}

Note. The number of sources after the each selection cut starting from the parent sample to the final one used in this study. The details are summarized at Sections 2.1.2 and 2.2.

data covering the optical, IR, and radio band for the WERGS sample and derived physical parameters of host galaxies such as stellar mass and star formation rate as well as the AGN luminosity estimated from the mid-IR bands. By using this data set, we will explore the AGN and host-galaxy properties of the ERGs. Throughout this paper, we adopt the same cosmological parameters as Yamashita et al. (2018) and Toba et al. (2019); $H_{0}=70 \mathrm{~km} \mathrm{~s}^{-1} \mathrm{Mpc}^{-1}, \Omega_{\mathrm{M}}=0.27$, and $\Omega_{\Lambda}=0.73$.

\section{Sample Selection and Properties}

Our initial sample is based on the WERGS sample ( $~ 3600$ sources; Yamashita et al. 2018), which compiled the HSC Subaru Strategic Program (HSC-SSP) optical counterparts of the VLA/FIRST data. Here, we briefly summarize the WERGS sample and the reader should refer to Yamashita et al. (2018) for the WERGS catalog and Toba et al. (2019) for the IR catalog of the WERGS sample. We also describe further selection criteria imposed on the sample, which are suitable for this study. The number of WERGS sources after each sample selection cut is also summarized in Table 1.

\subsection{WERGS Sample}

\subsubsection{VLA/FIRST}

The VLA/FIRST survey contains radio imaging data at $1.4 \mathrm{GHz}$ with a spatial resolution of 5!" 4 (Becker et al. 1995; White et al. 1997), which completely covers the footprint of the HSC-SSP wide-layer (see the text below). Yamashita et al. (2018) utilized the final release catalog of FIRST (Helfand et al. 2015) with the flux limit of $>1 \mathrm{mJy}$, and extracted 7072 FIRST sources in the HSC-SSP footprint.

\subsubsection{Subaru/HSC-SSP Data}

The HSC-SSP is an ongoing wide and deep imaging survey covering five broadband filters $(g, r, i, z$, and $y$ band; Aihara et al. 2018a; Bosch et al. 2018; Furusawa et al. 2018; Kawanomoto et al. 2018; Komiyama et al. 2018; Huang et al. 2018), consisting of three layers (wide, deep, and ultra deep). Yamashita et al. (2018) utilized the wide-layer S16A data release, which contains 
observations from 2014 March to 2016 January with a field coverage of $154 \mathrm{deg}^{2}$ (based on six fields; XMM-LSS, GAMA09H, WIDE12H, GAMA15H, HECTOMAP, and VVDS), and forced photometry of $5 \sigma$ limiting magnitude down to $26.8,26.4,26.4,25.5$, and 24.7 for the $g, r, i, z$, and $y$ bands, respectively (Aihara et al. 2018b). The average seeing in the $i$ band is $0 . " 6$, and the astrometric root-mean-squared uncertainty is about 40 mas. After removing spurious sources flagged by the pipeline, Yamashita et al. (2018) cross matched the FIRST sources with a search radius of $1^{\prime \prime}$. Yamashita et al. (2018) also required detections with $\mathrm{S} / \mathrm{N}>5$ in the $r, i$, and $z$ bands to qualify as an optical counterpart. This initial sample of Yamashita et al. (2018) comprised 3579 sources.

We then applied additional cuts to this sample. To remove radio galaxies from the initial WERGS sample whose radio emission might be dominated by the star formation of host galaxies and not by the AGNs, we set a lower-limit to the radio luminosity of $L_{1.4 \mathrm{GHz}}>10^{24} \mathrm{WHz}^{-1}$, which is equivalent to the radio emission from the host galaxies with a star formation rate of $\mathrm{SFR} \approx 250 M_{\odot} \mathrm{yr}^{-1}$ (Condon 1992; Condon et al. 2013). This cut is also supported by a steep decline in the radio luminosity function of starburst galaxies above $L_{1.4 \mathrm{GHz}}=$ $10^{23} \mathrm{WHz}^{-1}$ (e.g., Kimball et al. 2011; Padovani 2016; Tadhunter 2016). This criterion reduces the sample size to 3147.

Next, we limit our sample to compact sources in the radio band. This is important in order to reduce false optical identification by mismatching the optical sources with the locations of spatially extended radio-lobe emission. Yamashita et al. (2018) discussed this and categorized radio compact sources using the ratio of the total integrated radio flux density to the peak radio flux density $f_{\text {int }} / f_{\text {peak }}$. They treated the source as compact if the ratio fulfilled either of the two following equations,

$$
\begin{gathered}
f_{\text {int }} / f_{\text {peak }}<1+6.5 \times\left(f_{\text {peak }} / \mathrm{rms}\right)^{-1} \\
\log \left(f_{\text {int }} / f_{\text {peak }}\right)<0.1
\end{gathered}
$$

where $f_{\text {peak }} / \mathrm{rms}$ is the $\mathrm{S} / \mathrm{N}$ and $\mathrm{rms}$ is a local rms noise in the FIRST catalog, and the above two equations are obtained from the study by Schinnerer et al. (2007; see also the original criterion in Ivezić et al. 2002). We set an additional conservative requirement that there shall be no FIRST source in the surrounding $1^{\prime}$ (corresponding to $\lesssim 500 \mathrm{kpc}$ at $z \sim 1$ ) to select the isolated radio core emission and avoid coincident matching with radio lobes of other nearby FIRST sources. This reduces the sample to 2583 sources.

A further 10 sources were removed in crowded regions of the HSC footprint due to multiple HSC detections within $<1^{\prime \prime}$ of the HSC counterpart, bringing the sample down to 2573 sources.

Finally, we restricted the sample to sources with spectroscopic redshift $\left(\right.$ spec- $\left.z ; z_{\text {spec }}\right)$ or reliable photometric redshift (photo- $z$; $z_{\text {phot }}$ ) values. The spec- $z$ were obtained from SDSS DR12 (Alam et al. 2015), the GAMA project DR2 (Driver et al. 2011; Liske et al. 2015), and WiggleZ Dark Energy Survey project DR1 (Drinkwater et al. 2010). In this study, we limit the sample to the spec- $z$ range $0.3<z_{\text {spec }}<1.6$ to cover the same redshift range as the photo- $z$ sample. For the sources without spec- $z$, we utilized the photo- $z$ estimated using the Mizuki SED-fitting code which is one of the standard photo- $z$ packages for the HSC-SSP survey. The method utilizes the photometries of the five HSC-SSP bands (see
Tanaka 2015 and Tanaka et al. 2018, for more details) for the SED fitting. Yamashita et al. (2018) discussed that the HSC-SSP photo- $z$ derived by MIZUKI is reliable for $z_{\text {phot }}<1.6$ based on comparison with spectroscopic redshift in the COSMOS field. In addition, the redshifts of sources with $z_{\text {phot }}<0.3$ are sometimes erroneous because they lack the Balmer-break tracer in the HSC optical bands. Therefore, we limit the sample to $0.3<z_{\text {phot }}<1.6$. We further imposed requirements based on the reliable photo- $z$ fitting quality (reduced $\chi^{2}<3, \sigma / z_{\text {phot }}<0.2$, and $\sigma /\left(1+z_{\text {phot }}\right)<0.1$ ), following Toba et al. (2019). The resulting WERGS sample contains 1770 sources. Given that our sample largely relies on photo- $z$ results, we further discussed how possible erroneous photo$z$ might affect our main results in Appendix A.

\subsection{WERGS IR Catalog}

To study the AGN and host-galaxy properties in WERGS, Toba et al. (2019) compiled optical, near-IR, mid-IR, far-IR, and radio data for the WERGS sample. Toba et al. (2019) performed spectral energy distribution (SED) fitting with CIGALE (Boquien et al. 2019) and inferred physical properties, including the IR luminosity contributed from AGNs (IR AGN luminosity; $\left.L_{\mathrm{AGN}, \mathrm{IR}}\right)$, dust-extinction-corrected stellar mass $\left(M_{\star}\right)$, and SFR estimated from the decomposed host-galaxy IR emission. ${ }^{14}$ For the estimation of $L_{\mathrm{AGN}, \mathrm{IR}}$, Toba et al. (2019) considered the contamination of synchrotron radiation in the IR bands by extrapolating the power law from the radio bands. Thus, $L_{\mathrm{AGN}, \mathrm{IR}}$ is obtained purely from the dust emission heated by AGNs. The bolometric AGN luminosity is estimated by using the conversion $L_{\mathrm{AGN}, \text { bol }} \simeq 3 \times L_{\mathrm{AGN}, \mathrm{IR}}$ (Delvecchio et al. 2014; Inayoshi et al. 2018). Because Toba et al. (2019) restricted themselves to objects in an area of $\sim 95 \mathrm{deg}^{2}$, in which multi-wavelength information from $u$ band to far-IR was available, our sample is reduced to 1055 sources.

Some of the selected sources have intensive star formation rates, reaching $\mathrm{SFR} \approx 250 M_{\odot} \mathrm{yr}^{-1}$, so we applied an additional cut to remove possible star-formation-dominated radio galaxies using the ratio of IR and radio luminosity $\left(q_{\mathrm{IR}}\right.$; Helou et al. 1985; Ivison et al. 2010) defined by

$$
q_{\mathrm{IR}}=\log \left(\frac{L_{\mathrm{IR}} / 3.75 \times 10^{12}}{L_{1.4 \mathrm{GHz}}}\right),
$$

where $L_{\mathrm{IR}}$ is the total IR luminosity in units of $\mathrm{W}$ derived from CIGALE and $3.7 \times 10^{12}$ is the frequency in $\mathrm{Hz}$ corresponding to $80 \mu \mathrm{m}$, which makes $q_{\mathrm{IR}}$ a dimensionless quantity. We set $q_{\mathrm{IR}}<1.68$ (Del Moro et al. 2013) to select radio-excess (meaning jet-emission-dominated) sources. As a result, our final sample reduces to 1026 sources, and the resulting final number of ERGs fulfilling $\log \mathcal{R}_{\text {rest }}>4$ is 39 sources; they are shown as orange stars in Figure 1. We refer to the remaining 987 WERGS sources with $1<\log \mathcal{R}_{\text {rest }}<4$ as "normal radio galaxies (NRGs)" (shown with blue circles in Figure 1) hereafter.

Table 1 shows the number of sources for the full WERGS sample and the ERGs after each selection cut. ERGs suffer from the photo- $z$ quality cut more than the full WERGS sample because ERGs contain a relatively large fraction of the optically faintest sources with $i_{\mathrm{AB}}>25$, which sometimes makes the reliable photo- $z$ estimation difficult. The IR selection

\footnotetext{
14 The catalog containing physical parameters of Toba et al. (2019) is available at http://vizier.u-strasbg.fr/viz-bin/VizieR?-source=J/ApJS/243/15.
} 

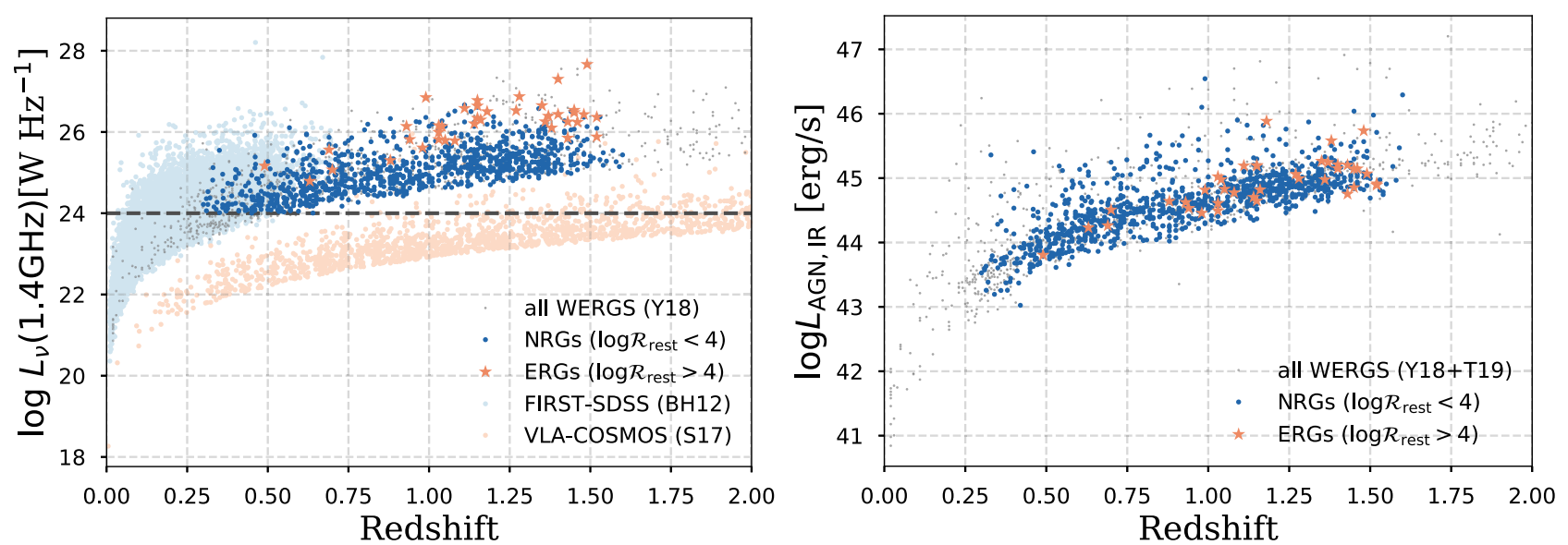

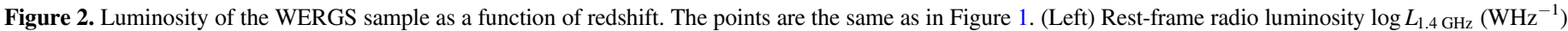

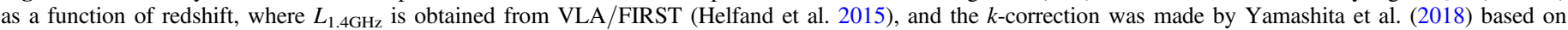

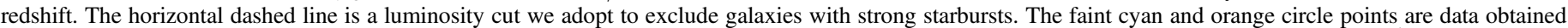

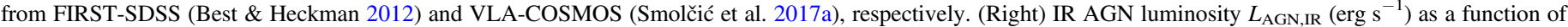

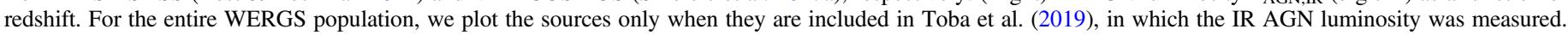

cut is another main factor in reducing the number of sources. This is mainly because the region available for the IR data is limited in the area of $\sim 95 \mathrm{deg}^{2}$ out of the $154 \mathrm{deg}^{2}$ (Toba et al. 2019). Note that this IR selection reduces the similar fraction of sources for the full WERGS sample (60\%) and ERGs (55\%).

\section{Results}

We summarize the properties of the obtained 39 ERGs with IR detections. First, we show the basic differences between ERGs and NRGs. Then, we show the properties of ERGs on SMBHs and host galaxies.

\subsection{Basic Sample Properties}

\subsection{1. $\log \mathcal{R}_{\text {rest }}$ versus g-band Magnitude}

Figure 1 shows the distribution of the radio-loudness parameter $\mathcal{R}_{\text {rest }}$ as a function of the rest-frame $g$-band magnitude $\left(g_{\mathrm{AB}}\right)$. The vertical dashed line at $g_{\mathrm{AB}}=22$ indicates the SDSS magnitude limit (e.g., Ivezić et al. 2002). Since most ERGs are fainter than the SDSS limit, Subaru/HSC has enabled us to investigate this unique population for the first time. Figure 1 also shows that most ERGs are very faint in the optical, with median magnitudes of $\left\langle g_{\mathrm{AB}}\right\rangle=24.5$. The current $8 \mathrm{~m}$ class telescopes are sufficiently sensitive to obtain spec- $z$ for the bright end of the ERGs sample down to $g_{\mathrm{AB}} \approx 24.0$ and with $30 \mathrm{~m}$ class telescopes, spec- $z$ can be obtained for sources down to $g_{\mathrm{AB}} \approx 26.0$.

\subsubsection{L-z Plane}

Figure 2 shows the radio and IR AGN luminosities of our sample as a function of redshift. The $1.4 \mathrm{GHz}$ radio luminosity $\left(L_{1.4 \mathrm{GHz}} \mathrm{W} \mathrm{Hz}^{-1}\right)$ is taken from Yamashita et al. (2018) or Toba et al. (2019), in which the integrated flux density $\left(f_{\text {int }}\right)$ obtained by the VLA/FIRST final catalog (Helfand et al. 2015) is used, and $k$-correction is applied by assuming a power-law radio spectrum with $f_{\nu} \propto \nu^{\alpha}$, where $\alpha$ is estimated with $\alpha=$ $\log \left(f_{1.4 \mathrm{GHz}} / f_{150 \mathrm{MHz}}\right) / \log \left(\nu_{1.4 \mathrm{GHz}} / \nu_{150 \mathrm{MHz}}\right)$ for the objects having TGSS $150 \mathrm{MHz}$ data (Intema et al. 2017) and $\alpha=-0.7$ for all others (e.g., Condon 1992).

As shown in the left panel of Figure 2, ERGs show relatively high radio luminosities with a median of $\left\langle\log \left(L_{1.4 \mathrm{GHz}}\right\rangle\right.$
$\left.\left.\mathrm{W} \mathrm{Hz}{ }^{-1}\right)\right\rangle=26.3$, which is one order of magnitude higher than that of the NRGs $\left(\left\langle\log \left(L_{1.4 \mathrm{GHz}} / \mathrm{W} \mathrm{Hz}^{-1}\right)\right\rangle=25.1\right)$. However, in terms of the IR AGN luminosity shown in the right panel, there is no clear difference between ERGs and the NRGs $\left\langle\log \left(L_{\mathrm{AGN}, \mathrm{IR}} / \mathrm{erg} \mathrm{s}^{-1}\right)\right\rangle=44.9$ for ERGs and $\left\langle\log \left(L_{\mathrm{AGN}, \mathrm{IR}} / \mathrm{erg} \mathrm{s}^{-1}\right)\right\rangle=44.6$ for the NRGs. We therefore assume ERGs to be intrinsically radio-louder sources than NRGs and we will discuss this in Section 4.1.

\subsection{Host-galaxy Properties}

The left panel of Figure 3 shows the distribution of our RGs in the SFR and $M_{\star}$ plane, where both values are obtained from the optical+IR SED fitting with CIGALE (Toba et al. 2019). It is known that most star-forming galaxies follow the mainsequence (MS) whose normalization moves upward with redshift, as galaxies are more gas-rich at higher $z$ (e.g., Brinchmann et al. 2004; Noeske et al. 2007; Elbaz et al. 2011; Whitaker et al. 2012; Schreiber et al. 2015). Above the MS, galaxies are referred to as starburst galaxies, producing stars more efficiently than regular star-forming galaxies. Pearson et al. (2018) measured the SFR and $M_{\star}$ using multi-wavelength data from UV to far-IR employing the CIGALE code, and their MS is delineated with the shaded area in Figure 3, whose bottom and top boundaries correspond to redshifts of $z=0.3$ and $z=1.6$ with the scatter of $\sigma=0.35$ at $z=0.3$ and $\sigma=0.24$ at $z=1.6$, respectively. In this study, we define the sources as starburst galaxies if they are located above the yellow shaded MS area, which is roughly consistent with the criteria in previous studies (e.g., Elbaz et al. 2011).

Considering that our sample spans a wide redshift range in $0.3<z<1.6$ and their rapid redshift evolution of MS, redshift dependence is a key factor to understanding the relation in the left panel of Figure 3. Therefore, we also calculate the ratios between the observed SFRs and those predicted from the MS relations at a given stellar-mass, that is, SFR/SFR(MS), and its redshift dependence are shown in the right panel of Figure 3. We also compile how the SFR and $M_{\star}$ relation evolves with redshift in Appendix B and Figure 10 (top panels).

Figure 3 shows several sequences of sources, notably at around sSFR $\sim 10^{-10.5}, \sim 10^{-9}$, and $\sim 10^{-8} \mathrm{yr}^{-1}$, and the sources locate scarcely between the sequences. This does not originate from the 

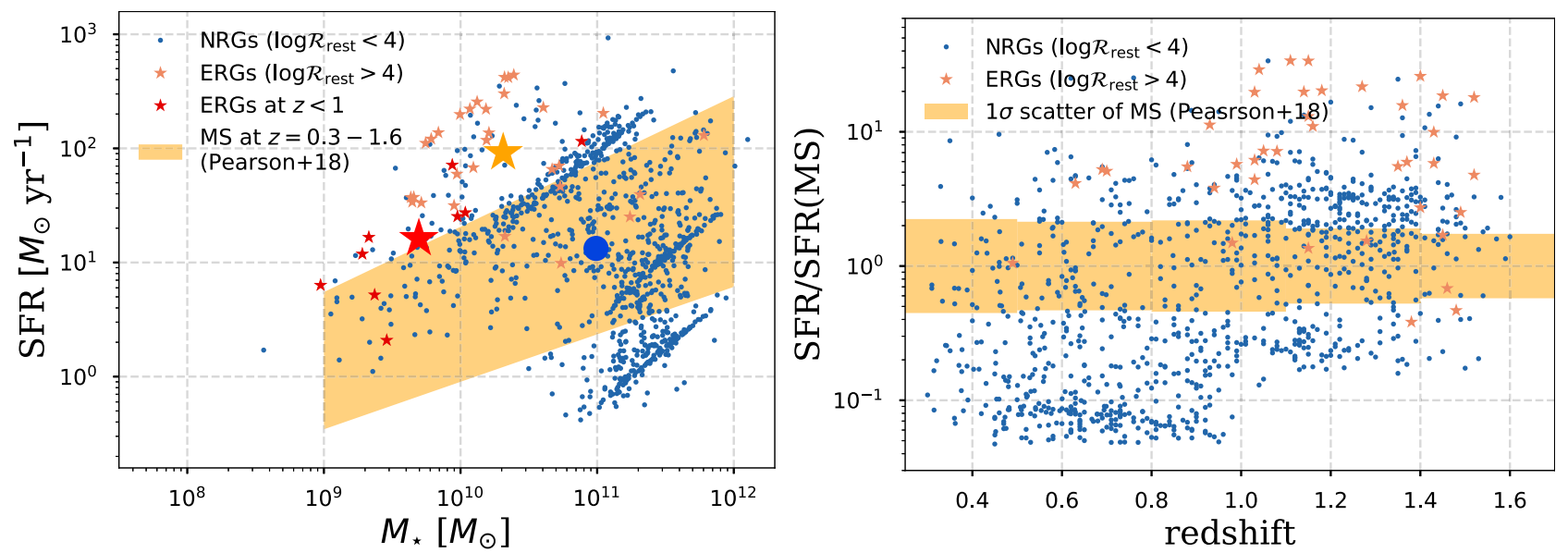

Figure 3. (Left) The relation between SFR and stellar mass $\left(M_{\star}\right)$ of the WERGS sample. Both values are obtained from the SED fitting by Toba et al. (2019). The blue circles represent the NRGs. The orange and red stars represent ERGs at $z>1$ and $z<1$, respectively. The large points show the average of each sample. The shaded area is the expected main-sequence region from $z=0.3$ (minimum) to $z=1.6$ (maximum) with the scatter of $\sigma=0.35$ at $z=0.3$ and $\sigma=0.24$ at $z=1.6$, which is obtained from Pearson et al. (2018). The redshift evolution of the relation is summarized in Figure 10. (Right) Redshift dependence of the ratio SFR/SFR(MS) of the WERGS sample. The $1 \sigma$ scatter is shown with the orange shaded area, which is also obtained from Pearson et al. (2018).

nature properties, but more mainly from the complications of the limited bin numbers of the SED fitting parameters of star-forming history (SFH). In our case, the most notable contribution is considered to be $f_{\text {burst }}$, the mass fraction of the burst population. Toba et al. (2019) set a limited parameter binning of $f_{\text {burst }}=0.001$, 0.1 , and 0.3 , which roughly corresponds to the bindings at sSFR $\sim 10^{-10.5}, 10^{-9}$, and $10^{-8} \mathrm{yr}^{-1}$. This binding might disappear once we follow the same SFH parameter binnings of Pearson et al. (2018), which was impossible for our studies because of the additional parameters of AGN and radio components which increase the computational time exponentially. Therefore, instead we assume that SFR in this study has a large uncertainty of $\sim 0.5$ dex. Please see the more detailed discussion on the choice of the SFH parameters and its affects on the SFR estimations (e.g., Schreiber et al. 2015; Ciesla et al. 2017; Pearson et al. 2018).

Although our SFR estimation by Toba et al. (2019) might harbor large uncertainties as discussed above, Figure 3 shows three important suggestions. One is that most ERGs are distributed above the MS by a factor of $\sim 10$ (see the right panel), suggesting that most ERGs are in starburst mode and they contain a copious amount of gas. This is indirect evidence for the availability of a cold gas supply fueling both star formation and the central SMBHs. The specific star formation rate defined by $\mathrm{SSFR}=\mathrm{SFR} / M_{\star}$ reaches $\mathrm{SSFR} \sim 10^{-8} \mathrm{yr}^{-1}$ for ERGs, suggesting that ERGs are in a rapid stellar-mass assembly phase with mass doubling times of only $\sim 100 \mathrm{Myr}$. This value is also comparable to one starburst duration (e.g., McQuinn et al. 2010) and thus most of the stellar component is expected to be dominated by a very young population.

Second, the onset of AGN feedback on the host galaxies has apparently not (yet) happened to most ERGs considering their location above the MS. This indicates a different picture from the conventional local RGs, which show a preference for massive host galaxies $\left(M_{\star}>10^{11} M_{\odot}\right)$ and fall below the MS because their strong AGN feedback quenches star formation (e.g., Seymour et al. 2007). Note that our sample requires IR detections for the CIGALE SED fitting to derive stellar mass and SFR, therefore, the WERGS not detected in IR do not appear in Figure 3. In addition to that, our sample requires radio-compact morphologies, which removes a large population of local, radio-extended RGs. These two selection criteria may introduce a selection bias since it is possible that these IR non-detection or radio-extended sources are clustered below the SF main sequence. It is nonetheless intriguing that ERGs are located at the top edge of the distribution within the WERGS sample with IR detections.

The third point is that, if we limit ourselves to the sources with $z<1$ (red star points), most of these are clustered at the low $-M_{\star}$ end, typically at $\log M_{\star} / M_{\odot}<10$. This suggests that the low- $z$ subsample of ERGs is potentially important with regard to the study of $\mathrm{BH}$ seeds. Considering that all of our sources fulfill (1) $L_{1.4 \mathrm{GHz}}>10^{24} \mathrm{WHz}^{-1}$, that is, they lie above the threshold where their radio luminosity could be produced by star formation, as discussed in Section 2.1.2, and (2) the radio-excess selection with $q_{\mathrm{IR}}<1.68$, their radio emission can only be achieved by the AGN jet, requiring the existence of an $\mathrm{SMBH}$ at the center. Therefore, ERGs contain massive $\mathrm{BH}$ candidates residing in low-mass galaxies.

Figure 4 shows the distribution of ERGs and NRGs in the $M_{\star}$ and $L_{1.4 \mathrm{GHz}}$ plane and illustrates the third point above from a different aspect. ERGs populate regions of comparatively small stellar mass and high $L_{1.4 \mathrm{GHz}}$, showing that higher $\mathcal{R}_{\text {rest }}$ sources tend to have smaller $M_{\star}$ as well as higher $L_{1.4 \mathrm{GHz}}$. The combined results from above and from Figure 1 suggest that smaller $M_{\star}$ sources tend to be observed as optically fainter sources and are therefore observed as high $\mathcal{R}_{\text {rest }}$ sources. We will discuss this point later in Section 4.1.

\subsection{SMBH Properties: Relation between $s B H A R$ and $\mathrm{M}_{\star}$}

Considering that most ERGs are very faint $\left(\left\langle g_{\mathrm{AB}}\right\rangle=24.5\right)$ in optical, as shown in Figure 1 and Section 3.1.1, obtaining the $\mathrm{BH}$ virial mass from spectroscopy (e.g., McLure \& Dunlop 2004) is time-consuming and difficult even if they are type-1 AGNs. This means that the direct measurement of the Eddington ratio ( $\lambda_{\text {Edd }} \equiv L_{\mathrm{AGN}, \text { bol }} / L_{\mathrm{Edd}}$, where $L_{\mathrm{Edd}}$ is Eddington luminosity $\left.L_{\mathrm{Edd}} \simeq 1.26 \times 10^{38}\left(M_{\mathrm{BH}} / M_{\odot}\right) \mathrm{erg} \mathrm{s}^{-1}\right)$, is difficult at this stage. Instead, we investigate the SMBH properties through the specific black hole accretion rate (hereafter, sBHAR), which may be considered a proxy for the Eddington ratio. The SBHAR is conventionally defined as $\mathrm{sBHAR}=L_{\mathrm{AGN}, \mathrm{bol}} / M_{\star} \mathrm{erg} \mathrm{s}^{-1} M_{\odot}^{-1}$ (e.g., Mullaney et al. 2012). The left panel of Figure 5 shows the 


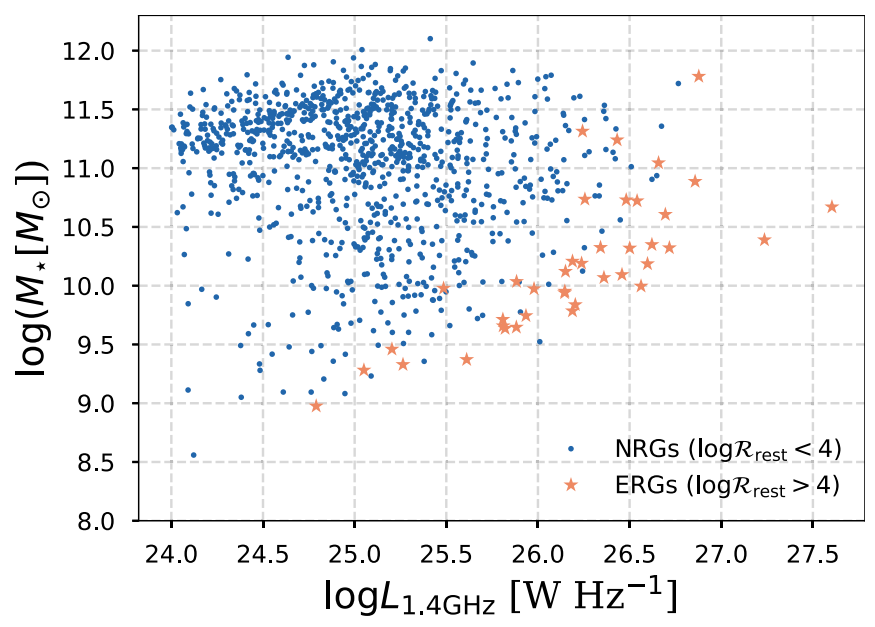

Figure 4. The stellar mass $\left(M_{\star}\right)$ as a function of the radio luminosity $\log L_{1.4 \mathrm{GHz}}$. The points are the same as in Figure 1.

distribution of sBHAR for ERGs and NRGs. The median sBHAR of ERGs is $\left\langle\log \left(\mathrm{sBHAR} / \mathrm{erg} \mathrm{s}^{-1} M_{\odot}^{-1}\right)\right\rangle=35.3$, which is an order of magnitude higher than that of the NRGs with $\left\langle\log \left(\mathrm{sBHAR} / \mathrm{erg} \mathrm{s}^{-1} M_{\odot}^{-1}\right)\right\rangle=34.0$. Based on Best \& Heckman (2012), the boundary of radiatively inefficient and efficient AGNs is at an Eddington ratio of $\log \lambda_{\text {Edd }} \sim-2$, which corresponds to $\log \left(\mathrm{sBHAR} / \mathrm{erg} \mathrm{s}^{-1} M_{\odot}^{-1}\right)=32-33$. Thus, almost all of our sources are considered to be radiatively efficient AGNs.

This difference in sBHAR between the two subgroups is more pronounced in the right panel of Figure 5, showing sBHAR as a function of $M_{\star}$. To illustrate the dependence of $M_{\star}$ and $\lambda_{\text {Edd }}$ on sBHAR more clearly, we use local scaling relations between $M_{\mathrm{BH}}$ and $M_{\star}$. Previous studies assumed a constant ratio of $M_{\mathrm{BH}} / M_{\text {bulge }}=(1.5-2.0) \times 10^{-3}$ (Marconi \& Hunt 2003; Häring \& Rix 2004) and $M_{\text {bulge }} \approx M_{\star}$ for simplicity (Aird et al. 2012, 2019; Mullaney et al. 2012; Delvecchio et al. 2018). In this study, we apply stellar-mass dependent values for $M_{\mathrm{BH}}$ with $M_{\mathrm{BH}} \propto M_{\star}^{\beta}$, where $\beta=1.4$ (KH13; Kormendy \& Ho 2013) and $\beta=1.05$ (RV15; Reines \& Volonteri 2015). With this relation, sBHAR can be written as a function of $\lambda_{\text {Edd }}$ and $M_{\star}$ by

$$
\begin{aligned}
& \operatorname{sBHAR}(\text { KH13 })=4.6 \times 10^{35} \lambda_{\text {Edd }}\left(M_{\star} / 10^{10} M_{\odot}\right)^{0.4}, \\
& \operatorname{sBHAR}(\mathrm{RV} 15)=3.2 \times 10^{34} \lambda_{\text {Edd }}\left(M_{\star} / 10^{10} M_{\odot}\right)^{0.05} .
\end{aligned}
$$

The right panel of Figure 5 shows that ERGs are more clustered in the high-Eddington-ratio regime. The expected median Eddington ratio by using Equation (4) is $\left\langle\log \lambda_{\text {Edd }}\right\rangle \approx-0.4$. Some sources appear to exceed the Eddington limit. NRGs cover a broader range in SBHAR and some massive galaxies with $M_{\star}>10^{11} M_{\odot}$ fall into the radiatively inefficient regime with $\lambda_{\text {Edd }}<-2$.

We caution that the above discussion does not take into account the redshift evolution of the $M_{\mathrm{BH}}-M_{\star}$ relation, which has been suggested in some studies (e.g., Woo et al. 2008; Merloni et al. 2010; Ding et al. 2020). In this case, the estimated $M_{\mathrm{BH}}$ becomes larger at a given $M_{\star}$, and the resulting Eddington ratio becomes smaller. Assuming the evolutional trend of Merloni et al. (2010), with $M_{\mathrm{BH}} / M_{\star}(z) \propto(1+z)^{0.68}$, and using a median redshift for the ERGs of $\langle z\rangle=1.1$, the normalization of sBHAR becomes slightly smaller by a factor of 1.6. As shown in Figure 5, this difference does not change our overall result. Although there are other significant uncertainties and possible systematics of the expected $\lambda_{\text {Edd }}$ values, under the basic assumptions of Equations (4) or (5), our results suggest that higher $\mathcal{R}_{\text {rest }}$ sources have higher sBHAR and ERGs might contain sources achieving super-Eddington accretion. We will discuss this point in Section 4.1.

In summary, our results indicate that ERGs exhibit high sBHAR with the expected Eddington ratio of $\left\langle\log \lambda_{\text {Edd }}\right\rangle \approx-0.4$ and some ERGs may be experiencing a super-Eddington phase. In addition, ERGs are starburst galaxies and their stellar mass is relatively lower than NRGs. ERGs appear to be in a phase preceding the onset of AGN feedback. If we limit the sample to $z<1$, most ERGs are low-mass galaxies with $M_{\star}<10^{10} M_{\odot}$. Therefore, lower- $z$ ERGs are also candidates of massive, rapidly growing BHs.

\section{Discussion}

\subsection{What Does High $\mathcal{R}_{\text {rest }}$ Mean?}

Figures 1 and 2 indicate that ERGs or high $\mathcal{R}_{\text {rest }}$ sources tend to trace optically faint but radio-loud AGNs. In addition, Figure 4 shows that higher $\mathcal{R}_{\text {rest }}$ tends to pick up relatively lower stellar-mass populations. This means that the selection of ERGs using $\mathcal{R}_{\text {rest }}$ has a preference of selecting smaller $M_{\star}$ and, therefore, $\mathcal{R}_{\text {rest }}$ using optical bands does not seem to trace AGN accretion disk emission in the optical band. This is not a new result, but it is a long-standing issue surrounding the use of the optical radio loudness $\mathcal{R}_{\text {rest }}$ : it is strongly affected by hostgalaxy contamination and/or the nuclear obscuration around the SMBHs (Terashima \& Wilson 2003; Ho 2008). This raises the question of whether ERGs are intrinsically radio-loud or not.

To investigate this, we estimate the "intrinsic" radio loudness $\mathcal{R}_{\text {int }}$; the energy balance between the jet and accretion-disk defined by $\mathcal{R}_{\text {int }}=L_{\text {jet }} / L_{\mathrm{AGN} \text {,bol }}$, which is frequency-independent and also free from the contamination of host-galaxy components unlike $\mathcal{R}_{\text {rest }}$ (e.g., Terashima \& Wilson 2003). For the accretion-disk luminosity, we use the bolometric AGN luminosity $L_{\mathrm{AGN}, \text { bol }}$ obtained from the IR AGN luminosity $\left(L_{\mathrm{AGN}, \mathrm{IR}}\right)$ since $L_{\mathrm{AGN}, \mathrm{IR}}$ is derived from IR SED decomposition, which traces the re-radiation of the dust powered by AGNs and is mostly insusceptible to absorption (Gandhi et al. 2009; Ichikawa et al. 2012, 2017, 2019; Asmus et al. 2015).

The total jet power of the AGNs $\left(L_{\text {jet }}\right)$ can be estimated from the radio luminosity of the compact core or from the total radio emission (Willott et al. 1999; Cavagnolo et al. 2010; O'Sullivan et al. 2011). We adopt the relation between $L_{\text {jet }}$ and $L_{1.4 \mathrm{GHz}}$ of Cavagnolo et al. (2010),

$$
L_{\text {jet }}=7.3 \times 10^{43}\left(L_{1.4 \mathrm{GHz}} / 10^{24} \mathrm{~W} \mathrm{~Hz}^{-1}\right)^{0.70} \mathrm{erg} \mathrm{s}^{-1} \text {. }
$$

Note that the above equation is derived with the assumption that most radio sources have a spectral index of $\alpha=-0.8$. This value is almost consistent with the median value of our WERGS sample; $\alpha=-0.65$ for the sources with detections at both TGSS $150 \mathrm{MHz}$ and VLA/FIRST $1.4 \mathrm{GHz}$ or the fiducial value of $\alpha=-0.7$ for the sources whose radio band detection is only in one band (i.e., VLA/FIRST 1.4 GHz only) (Condon 1992). Shabala \& Godfrey (2013) recently investigated the effect of the radio source size in the jet-power estimation and found the dependence on the source size to be $\propto D^{0.58}$, suggesting that the effect of the source size is small. Since our sample is selected only for radiocompact emission in VLA/FIRST with a spatial resolution of $\sim 5^{\prime \prime}$ 

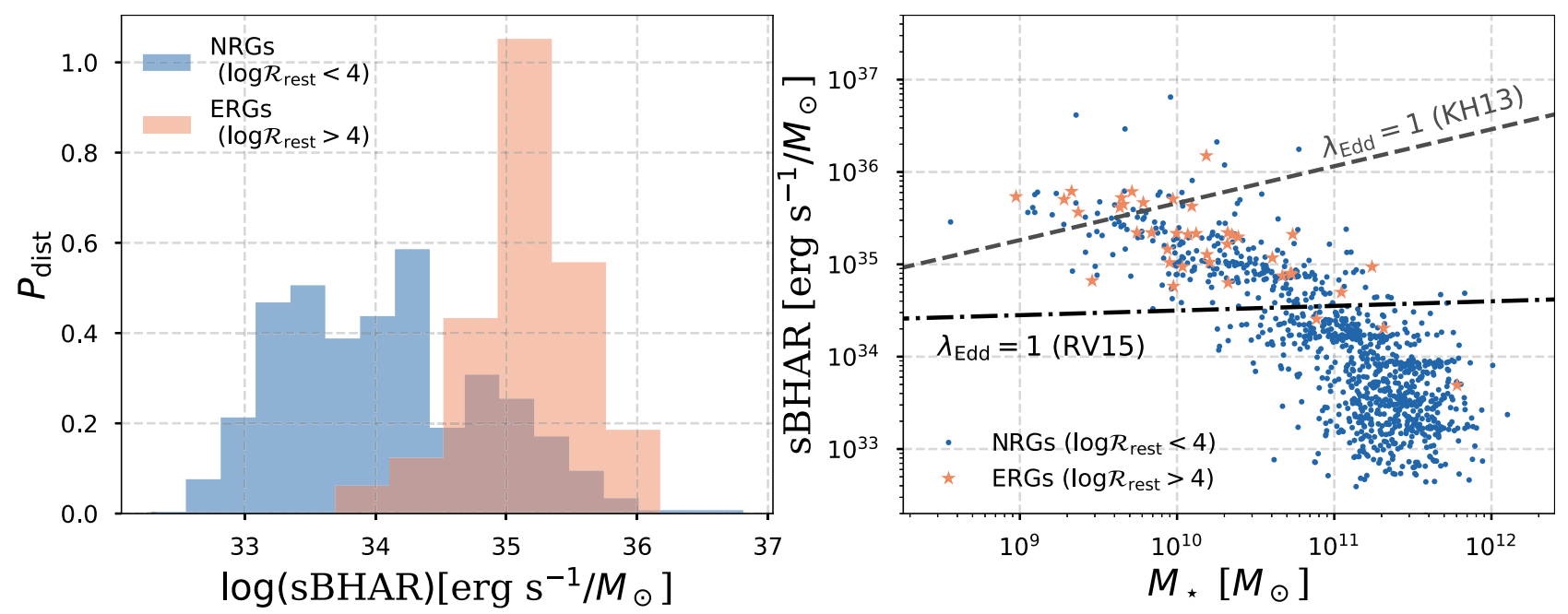

Figure 5. sBHAR properties for ERGs and NRGs in this study. The colors and symbols are same as in Figure 1. (Left) The distribution of sBHAR. The median of sBHAR is $\left\langle\log \left(\mathrm{sBHAR} / \mathrm{erg} \mathrm{s}^{-1} M_{\odot}^{-1}\right)\right\rangle=35.3$ for ERGs and $\left\langle\log \left(\mathrm{sBHAR} / \mathrm{erg} \mathrm{s}^{-1} M_{\odot}^{-1}\right)\right\rangle=34.0$ for NRGs. (Right) The relation between sBHAR and $M_{\star}$. The two straight lines are the expected Eddington limits $\lambda_{\mathrm{Edd}}=1$ using Equation (4) (gray dashed line) and using Equation (5) (black dotted-dashed line). The redshift evolution of the relation is summarized in the middle panels of Figure 10.
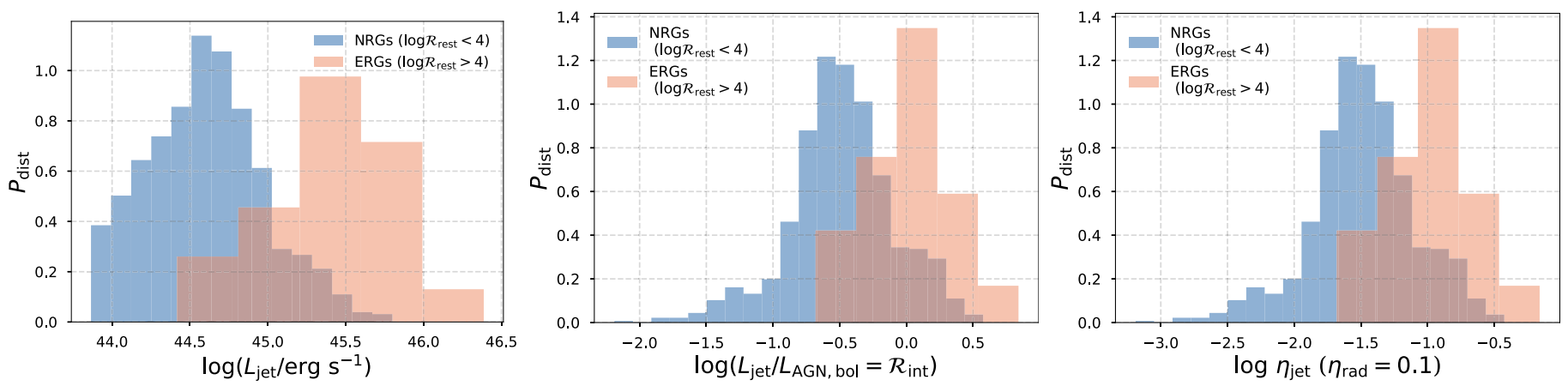

Figure 6. The distribution of $L_{\text {jet }} / \mathrm{ergs}^{-1}$ (left), $L_{\text {jet }} / L_{\mathrm{AGN}, \text { bol }}$, an indicator of intrinsic $\mathcal{R}_{\text {int }}$ (middle) and $\eta_{\text {jet }}$ assuming the radiative efficiency of 0.1 (right). The colors are the same as in Figure 1.

(or $\lesssim 20 \mathrm{kpc}$ at $z \sim 1$ ) this again mitigates the size dependence; otherwise, the sources are ultra-compact sources.

The left panel of Figure 6 shows the distribution of $L_{\text {jet }}$, spanning $44.0<\log \left(L_{\text {jet }} / \mathrm{erg} \mathrm{s}^{-1}\right)<46.4$ and the median values are $\left\langle\log L_{\text {jet }} / \mathrm{erg} \mathrm{s}^{-1}\right\rangle=45.4$ for ERGs and $\left\langle\log L_{\text {jet }} / \mathrm{erg} \mathrm{s}^{-1}\right\rangle=44.6$ for NRGs, which is equivalent to the radio-loud quasar level (e.g., Best et al. 2005; Inoue et al. 2017). This is naturally expected since we apply the simple conversion relation (Equation. 6). All of our sources are selected based on VLA/FIRST detection with the $>1$ mJy flux limit and the radio luminosity range is similar to that of the FIRST-SDSS sample, although they are located in relatively higher- $z$ (see Figure 2).

This high $L_{\mathrm{jet}}$ is powerful enough to produce a radio jet with a size of $\gtrsim 10 \mathrm{kpc}$ that can disperse the interstellar medium (e.g., Nesvadba et al. 2017) and create cavities in the galactic interstellar medium and larger scale intergalactic environment to possibly quench star formation in host galaxies (e.g., McNamara \& Nulsen 2007). Given the compact radio sizes of the sources in our sample $\left(<5^{\prime \prime}\right.$ or $\lesssim 20 \mathrm{kpc}$ at $\left.z \sim 1\right)$ and given the fact that the host galaxies are still in an active star-forming phase (Figure 3), ERGs and most WERGS sources above or on the SF main sequence may still be in the phase before the onset of huge kinetic radio feedback.
The middle panel of Figure 6 shows the distribution of $\mathcal{R}_{\text {int }}$ and the median values of the two populations are $\left\langle\log \mathcal{R}_{\text {int }}\right\rangle=0.0$ for ERGs, and $\left\langle\log \mathcal{R}_{\text {int }}\right\rangle=-0.5$ for NRGs. For half of the ERGs, $\mathcal{R}_{\text {int }}>1$, that is, the jet kinetic power is higher than the radiation from the accretion disk. The physical properties of higher $\mathcal{R}_{\text {rest }}$ sources are more clearly illustrated in Figure 7, which shows the distribution of ERGs and other WERGS sources in the $\mathcal{R}_{\text {int }}{ }^{-}$ sBHAR plane. The yellow shaded area is the region corresponding to $\lambda_{\mathrm{Edd}}=1$ assuming Equation (4) for the range of $8<\log \left(M_{\star} / M_{\odot}\right)<12$. The other WERGS sources are shown with a color gradation in $\log \mathcal{R}_{\text {rest }}$, with higher $\mathcal{R}_{\text {rest }}$ lying toward the top right of the plot. This means that higher $\mathcal{R}_{\text {rest }}$ sources tend to have both higher $\lambda_{\text {Edd }}$ and higher $\mathcal{R}_{\text {int }}$. It is well known that radio galaxies are more radio-loud as the Eddington-scaled accretion rate decreases, producing the sequence from the top left region toward the bottom right one in Figure 7 (Ho 2002; Merloni \& Heinz 2007; Panessa et al. 2007; Sikora et al. 2007; Sikora et al. 2013; Ho 2008), and overall NRGs also follow this trend. However, ERGs are located in the top right part of the plane, suggesting that ERGs contain rapidly growing $\mathrm{BH}$ in the center, and at the same time harbor powerful, compact radio jets.

These radio galaxies are different from the conventional radio galaxies in the local universe (Seymour et al. 2007). Delvecchio et al. (2018) recently found that high- $z(z>1.5)$ radio galaxies discovered in the VLA/COSMOS survey tend to 


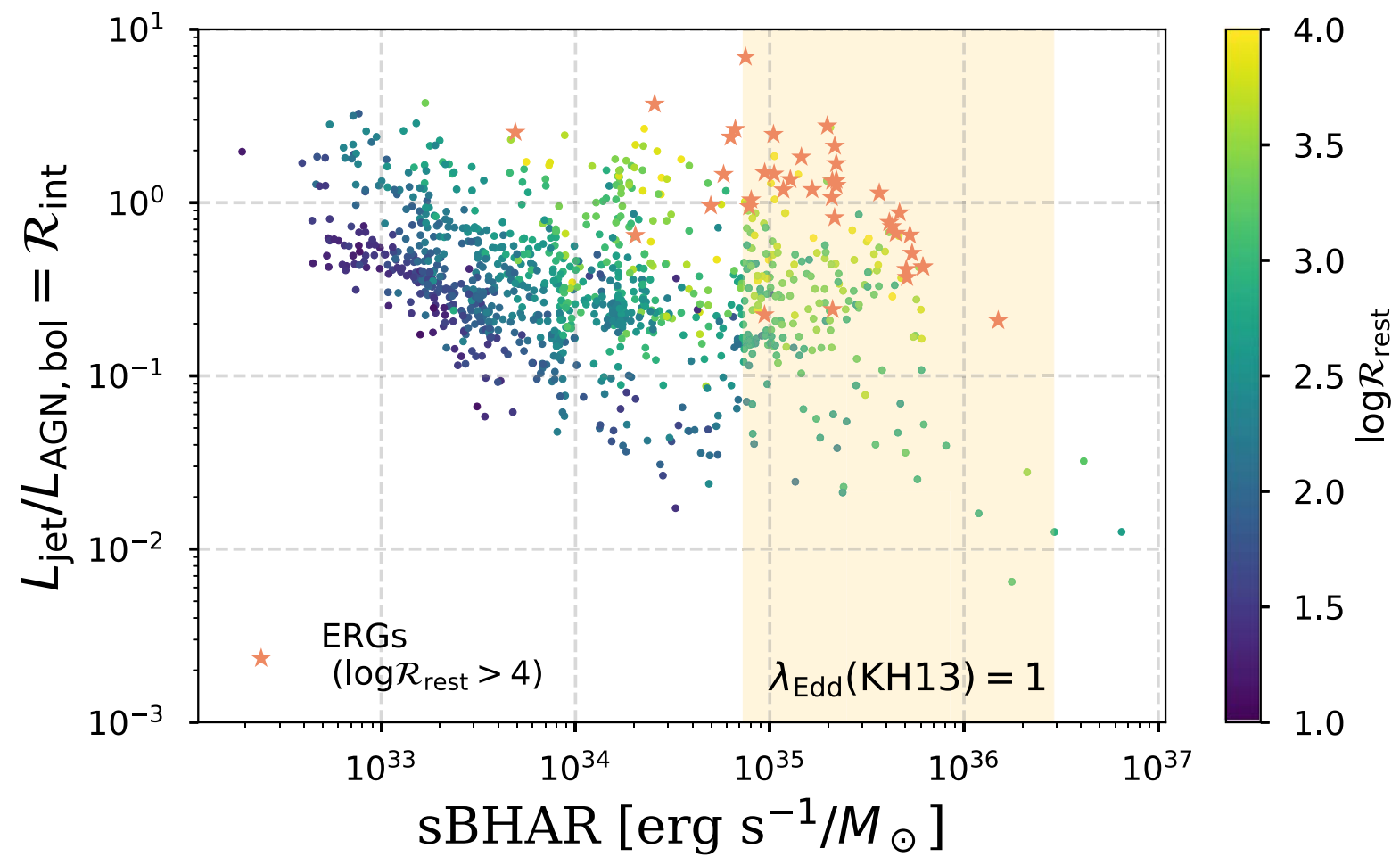

Figure 7. The relationship between $L_{\mathrm{jet}} / L_{\mathrm{AGN}, \text { bol }}=\mathcal{R}_{\text {int }}$ and sBHAR. The yellow shaded area represents the corresponding $\lambda_{\mathrm{Edd}}=1$ region assuming that $M_{\mathrm{BH}}$ is based on the $M_{\mathrm{BH}}-M_{\star}$ relation by Kormendy \& Ho (2013). The symbols are same as those in Figure 1, but other WERGS sources are shown with color gradation based on $\mathcal{R}_{\text {rest }}$ in the range of $1<\log \mathcal{R}_{\text {rest }}<4$. The redshift evolution of the relation is summarized in the bottom panels of Figure 10.

have high Eddington ratios with $\log \lambda_{\text {Edd }}>-2$ and that they are also star-forming or starburst galaxies. This is consistent with the properties of our overall WERGS sample (see also Toba et al. 2019) and thus ERGs occupy the extreme end of highly accreting $\mathrm{BHs}$ in radio galaxies.

\section{2. $\eta_{j e t}$ and the Origin of Radio Emission in ERGs}

Figure 7 indicates that ERGs are a very interesting population, showing both radiative (high $\lambda_{\text {Edd }}$ ) and jet-efficient (high $\mathcal{R}_{\text {int }}$ ) emission, which were missed in previous surveys. To investigate the jet properties in more detail, we here discuss the jet efficiency of ERGs and compare the obtained values with other radio sources. The jet production efficiency is defined as

$$
\eta_{\text {jet }}=L_{\text {jet }} / \dot{M}_{\mathrm{BH}} c^{2}=\eta_{\text {rad }} L_{\text {jet }} / L_{\mathrm{AGN}, \mathrm{bol}}
$$

where $\eta_{\text {rad }}$ is the radiation efficiency of an AGN accretion disk, $c$ is the speed of light, and $\dot{M}_{\mathrm{BH}}$ is the mass accretion rate onto the SMBH through the disk. In the following, we adopt a canonical value of $\eta_{\mathrm{rad}}=0.1$ based on the Soltan-Paczynski argument (Soltan 1982).

The distribution of $\eta_{\mathrm{jet}}$ is shown in the right panel of Figure 6. The median values are $\left\langle\log \eta_{\text {jet }}\right\rangle=-1.0 \pm 0.3$ for ERGs and $\left\langle\log \eta_{\text {jet }}\right\rangle=-1.5 \pm 0.4$ for NRGs. These values are slightly higher or consistent with the nearby radio AGN population $\left(\log \eta_{\text {jet }} \sim-1.5\right)$ whose host galaxies are massive $\left(M_{\star}>10^{11} M_{\odot}\right.$; Nemmen \& Tchekhovskoy 2015). However, the local radio AGN population generally has much smaller Eddington ratios of $\log \lambda_{\text {Edd }}<-2$. This population would be located in the top left region in Figure 7, so they are clearly different from ERGs. The $\eta_{\text {jet }}$ of ERGs is higher by a factor of $\sim 10$ compared to the SDSS radio quasar population, whose radio jet efficiency lies at $\left\langle\log \eta_{\text {jet }}\right\rangle=-2.0 \pm 0.5$ (e.g., van Velzen \& Falcke 2013; Inoue et al. 2017). Considering that the SDSS radio quasars on average show $\log \lambda_{\text {Edd }} \sim-1$, those radio quasars would be located at the bottom center to bottom right of Figure 7, which is again different from the distribution of ERGs. Therefore, the disk-jet connection may be fundamentally different from the standard disk model, which conventionally describes the quasar/AGN accretion disk emission. One possible origin of this high production efficiency of radiation and jets is that the accretion disk of ERGs is actually in the "radiatively inefficient" state, but the physical origin of radiative inefficiency is different from the disks in the local radio galaxies. For example, the ERGs may be undergoing more rapid mass accretion (the so-called slim disk model; Abramowicz et al. 1988). Recent radiation hydrodynamical simulations suggest that when the mass accretion rate significantly exceeds the Eddington rate, radiation is effectively trapped within the accreting matter and advected to the central $\mathrm{BH}$ before escaping by radiative diffusion. As a result, the emergent radiation luminosity is saturated at the order of $L_{\text {Edd }}$ (i.e., $\eta_{\text {rad }} \lesssim 0.1$ at $\gg \dot{M}_{\text {Edd }}$ ) and the accretion flow turns into a radiatively inefficient state even with a superEddington accretion rate (e.g., Ohsuga et al. 2005, 2009; McKinney et al. 2014; Inayoshi et al. 2016; Takeo et al. 2020). This phase is considered to be a key process of the $\mathrm{BH}$ seed growth in the high- $z(z>6)$ universe to describe the already known massive high- $z$ SMBHs (e.g., Mortlock et al. 2011; Wu et al. 2015; Bañados et al. 2018; Onoue et al. 2019).

In the situation where the accretion rate is well above the Eddington rate, a relativistic jet can be launched by the BlandfordZnajek mechanism if large-scale magnetic fields exist in the innermost region (Blandford \& Znajek 1977; Tchekhovskoy et al. 2011). The jet behavior of these extreme cases has been 


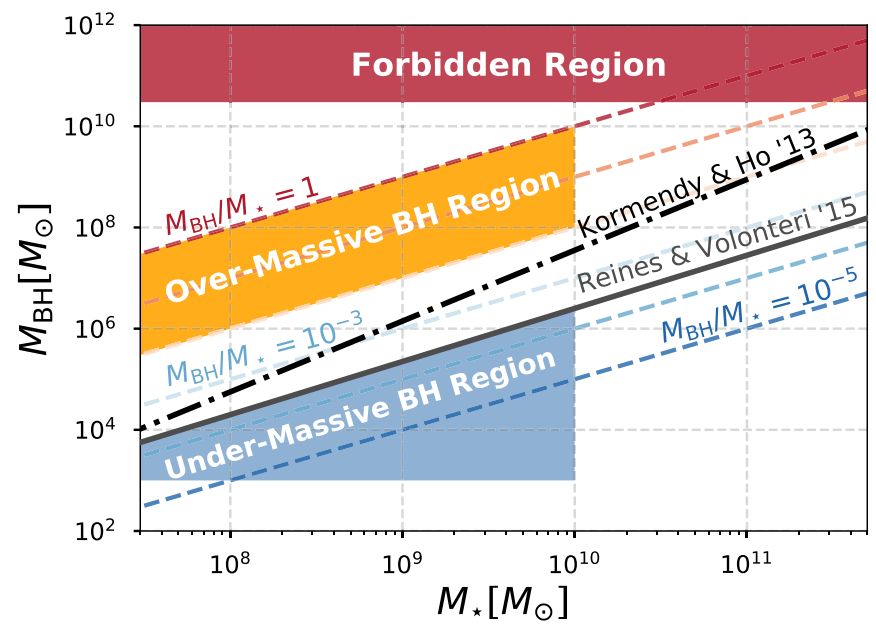

Figure 8. A schematic view of the relationship between $M_{\mathrm{BH}}$ and $M_{\star}$. The local relations by Kormendy \& Ho (2013) and Reines \& Volonteri (2015) are shown as dotted-dashed (black; the fiducial relation in this study) and solid-line (gray), respectively. The orange shaded region represents the range of overmassive BHs, which is at least one order of magnitude higher than the local relations (Case 1 in Section 4.3) and the region is delineated by $10^{8}<M_{\star} / M_{\odot}<10^{10}$ and $10^{-2}<M_{\mathrm{BH}} / M_{\star}<1$. The blue shaded region represents the range of undermassive BHs, in which host galaxies form first and BHs grow later (Case 2 in Section 4.3). This region is delineated by $10^{8}<M_{\star} /$ $M_{\odot}<10^{10}$ and $M_{\mathrm{BH}}>10^{3} M_{\odot}$ andsmaller than the local relation by Reines \& Volonteri (2015). The red shaded region represents the forbidden region where the $\mathrm{BH}$ cannot exceed $M_{\mathrm{BH}} \lesssim 3 \times 10^{10} M_{\odot}$ (Inayoshi \& Haiman 2016; Ichikawa \& Inayoshi 2017).

investigated by several authors (Sadowski et al. 2014; Sadowski \& Narayan 2015) using a 3D general relativistic radiation magnetohydrodynamical simulation (GRRMHD) code. They find that, in this (super-)Eddington accretion phase, BHs emit a significant amount of radiation and jet energy and both the radiation and jet power approach around the Eddington limit, which appears to be the case for ERGs and the sources in the top right region in Figure 7 (see also Blandford et al. 2019). Thus, the study of ERGs at $z \sim 1$ will be complementary to studies utilizing the next generation instruments capable of observing seed BHs in the early universe at $z \gtrsim 7$ (Haiman et al. 2019) in the investigations of the growth of seed BHs.

\subsection{Alternative Scenarios: If ERGs Do Not Follow Local Scaling Relations between $\mathrm{M}_{B H}$ and $\mathrm{M}_{\star}$}

The discussion related to the Eddington ratio depends on the $M_{\mathrm{BH}}$ estimation, which in turn relies on the local scaling relation of Kormendy \& Ho (2013). However, it is not obvious that this relation also holds in the low-mass end in which many ERGs probably reside. Although the current data cannot give any constraints on this issue and a more detailed study is beyond the scope of this paper, we discuss two alternative scenarios in which our galaxies are above (case one) or below (case two) the correlation between $M_{\mathrm{BH}}$ and $M_{\star}$ as shown in Figure 8 .

Case one reflects the possibility that ERGs might host overmassive BHs compared to the relation by Kormendy \& Ho (2013), with the BH-to-stellar-mass ratio $f_{\mathrm{b}, \star} \equiv M_{\mathrm{BH}} / M_{\star} \gtrsim 10^{-2}$ (see the orange shaded area in Figure 8). In this case, concerns surrounding super-Eddington accretion discussed in Sections 3.3 and 4.1 are alleviated: the median Eddington ratio for ERGs can be parameterized by $\left\langle\lambda_{\text {Edd }}\right\rangle \simeq 0.17\left(f_{\mathrm{b}, \star} / 0.01\right)^{-1}$.

In addition, the possible future growth path in the plane of $M_{\mathrm{BH}}$ and $M_{\star}$ can be discussed by assuming the current $\mathrm{BH} /$ stellar mass assembly rate. Since the radiation efficiency is $\eta_{\text {rad }}=0.1$ in the sub-Eddington regime, the $\mathrm{BH}$ accretion rate is estimated as $\dot{M}_{\mathrm{BH}}=L_{\mathrm{AGN}, \text { bol }} /\left(\eta_{\mathrm{rad}} c^{2}\right) \simeq 1.7 \times 10^{-3} f_{\mathrm{b}, \star}^{-1} \dot{M}_{\mathrm{Edd}}$, where the median Eddington ratio above is substituted (note that this equation is valid at $f_{\mathrm{b}, \star} \gtrsim 1.7 \times 10^{-3}$ ). Comparing the $\mathrm{BH}$ accretion rate to the $\mathrm{SSFR}$ of $\mathrm{ERGs}\left(\mathrm{sSFR}=\mathrm{SFR} / M_{\star} \simeq\right.$ $10^{-8} \mathrm{yr}^{-1}$; see Figure 3 ), we obtain $\dot{M}_{\mathrm{BH}} / \mathrm{SFR} \simeq 4 \times 10^{-3}$, which is independent of the choice of $f_{\mathrm{b}, \star}$. Therefore, the BHgalaxy mass ratio for ERGs is expected to approach a canonical value observed in the local universe (e.g., $f_{\mathrm{b}, \star} \sim 3 \times$ $10^{-3}$; Kormendy \& Ho 2013). This evolutional path might be analogous to that of known high- $z$ luminous quasars whose SMBHs are likely overmassive compared to the local relation with $10^{-2} \lesssim f_{\mathrm{b}, \star} \lesssim 2 \times 10^{-1}$ (e.g., Wang et al. 2013, 2016; Trakhtenbrot et al. 2015; Decarli et al. 2018).

Case two considers the possibility that ERGs might host very undermassive BHs with $f_{\mathrm{b}, \star} \lesssim 10^{-3}-10^{-4}$ as shown in the blue shaded region in Figure 8 . For instance, the overall normalization for late-type galaxies may be considerably lower than for the local relation of early-type galaxies (e.g., Reines \& Volonteri 2015; Läsker et al. 2016; Greene et al. 2020). This "galaxy grows first, BHs come later" phase may occur because gas accretion onto the BHs is quite inefficient due to efficient stellar feedback, e.g., star formation-driven outflows, and the shallow halo potential until the stellar-mass reaches a critical value of $M_{\star} \sim 10^{10.5} M_{\odot}$ (e.g., Bower et al. 2017; Habouzit et al. 2017). However, once the galaxy reaches the critical mass, above which star formation-driven outflows no longer prevent gas accretion onto the galactic nuclei, they abruptly switch to a rapid growth phase in the absence of feedback, and finally merge into the local relation. ERGs with $M_{\star} \gtrsim 10^{10.5} M_{\odot}$ might be in such a rapidly growing phase.

The scenario above looks promising but it is worth discussing the critical mass value because the stellar mass of most ERGs actually lies below the expected critical mass. Beside, Figure 5 shows that the median sBHAR (and therefore $\left.\lambda_{\text {Edd }}\right)$ tends to be higher for less massive ERGs with the median Eddington ratio of $\left\langle\lambda_{\text {Edd }}\right\rangle \simeq 1.7\left(f_{\mathrm{b}, \star} / 10^{-3}\right)^{-1}$, suggesting that the extreme $\mathrm{BH}$ growth is possible (at least temporally) even in lower mass galaxies. This discrepancy may reflect that there is a missing understanding about the physics of AGN feedback and gas feeding in low-mass galaxies.

Another interesting point in the second scenario is that the Eddington ratio for several ERGs becomes as high as $\lambda_{\text {Edd }} \sim 10^{2}$. Recent numerical simulations suggest that for a highly magnetized accretion disk around a rapidly spinning $\mathrm{BH}$, the disk transits into a magnetically arrested disk (MAD) state and produces high radiative luminosity (e.g., McKinney et al. 2014; Sadowski \& Narayan 2015). Through the emission process, the level of high luminosity could be achieved only when the $\mathrm{BH}$ mass accretion exceeds $\sim 10^{3} \dot{M}_{\text {Edd }}$ (Inayoshi et al. 2016; see also Figure 5 in Inayoshi et al. 2020). Therefore, if this scenario is true, future follow-up observations of those ERGs with such high Eddington ratios would reveal the properties of possibly hyperEddington accreting BHs (Takeo et al. 2020).

\subsection{Prospects for AGN Feedback in Low-mass Galaxies through ERGs}

In recent years, the theoretical study of AGN feedback has been extended to low-mass galaxies (Silk 2017). This was motivated mainly by the increasing number of intriguing observational results showing signs of AGN feedback in 
low-mass galaxies (Nyland et al. 2017; Penny et al. 2018; Dickey et al. 2019; Kaviraj et al. 2019; Mezcua et al. 2019). Our results show that the jet power of $L_{\text {jet }}>10^{44} \mathrm{erg} \mathrm{s}^{-1}$ in ERGs is equivalent to that produced from radio quasars and local radio AGNs residing in massive galaxies. This suggests the presence of a strong jet that can disperse or even expel the interstellar medium (e.g., Morganti et al. 2005; Holt et al. 2008; Nesvadba et al. 2017) or produce cavities in the host galaxies, which are often observed in the local massive counterparts (e.g., Rafferty et al. 2006; McNamara \& Nulsen 2007; Bîrzan et al. 2008; Blandford et al. 2019). On the other hand, low stellar-mass ERGs are still in a starburst phase, indicative of a substantial gas reservoir, and lacking clear signatures of negative AGN feedback. ${ }^{15}$

To investigate whether such a negative feedback from the jet is effective in the host galaxies, it is necessary to obtain high spatial resolution radio images that resolve the host galaxy down to scales of $<10 \mathrm{kpc}$ (Reines et al. 2020). Our sample of compact radio sources from VLA/FIRST with a spatial resolution of $\sim 5^{\prime \prime}$ only provide poor upper-bounds of the radio jet size of $<40 \mathrm{kpc}$ at $z \sim 1$. Future higher quality observations with subarcsecond resolution will provide more insight into the effect of the jet on the host galaxy.

Given that the $\mathrm{BH}$ mass of ERGs might reach the massive $\mathrm{BH}$ range below $M_{\mathrm{BH}} \sim 10^{6} M_{\odot}$, studying the properties of ERGs gives a window into understanding seed $\mathrm{BH}$ growth in the high- $z$ universe. Some observational studies recently suggested that AGN feedback might also have a positive effect on the host galaxies and intensive in situ star formation is ignited in the outflowing gas launched by the AGN (Maiolino et al. 2017). Recent numerical simulations of jet-driven feedback also predict star formation triggered on galaxy scales by the overpressure of the jet during the first few Myrs of strong interaction between the jet and the interstellar medium (Wagner \& Bicknell 2011; Gaibler et al. 2012; Wagner et al. 2016; Mukherjee et al. 2018). If the radio jets in low-mass galaxies have a positive impact on both star formation and $\mathrm{BH}$ growth, then radio-selected AGNs in low-mass galaxies are an intriguing population for understanding the $\mathrm{BH}$ and stellar mass assembly in the environment of shallow potential low-mass galaxies.

\subsection{Wide Area Radio and Optical Surveys: Potential for Discovering Previously Missed Populations}

The small number density of ERGs $\left(\sim 1\right.$ source $\left.\mathrm{deg}^{-2}\right)$ and their optical faintness of $i_{\mathrm{AB}} \sim 25$ suggest that ERGs were easily missed in previous optical surveys of radio sources. Our work shows that ERGs can be discovered with wide $(>100$ $\left.\operatorname{deg}^{2}\right)$ and deep $\left(i_{\mathrm{AB}} \lesssim 26\right)$ optical follow-up observations of radio sources. The number of known ERGs will increase in the near future, once the Subaru/SSP survey is complete, covering an area of $\sim 10^{3} \mathrm{deg}^{2}$. The upcoming Subaru/Prime Focus Spectrograph (PSF; Takada et al. 2014) will conduct extensive optical and near-IR spectroscopic follow-up observations in the footprint of the HSC-SSP field, providing spec- $z$ information of ERGs with $i_{\mathrm{AB}} \lesssim 24$. The forthcoming LSST survey (Ivezić et al. 2019) will cover half of the sky, and it will also increase the number of sources by another order of magnitude, resulting

\footnotetext{
${ }^{15}$ Numerical simulations suggest that mechanical feedbacks associated with jets would impact upon their host galaxies with a significant delay time, which might be marginally longer than the typical AGN lifetime (Costa et al. 2018).
}

in $\sim 10^{4}$ sources. A significant fraction of these ERGs will also be expected to reside in low-mass galaxies. Therefore, the combination of wide area (either shallow or deep) radio and deep optical imaging and spectroscopic surveys will give us a statistically large number of massive $\mathrm{BH}$ candidates in the coming years.

Massive BH searches have been conducted with multiwavelength data; optical spectroscopy using broad $\mathrm{H} \alpha$ emission (Greene \& Ho 2004, 2007; Xiao et al. 2011) or narrow emissionline ratios (e.g., Barth et al. 2008; Reines et al. 2013), mid-IR $(W 1-W 2)$ colors in WISE (Satyapal et al. 2014; Sartori et al. 2015; Secrest et al. 2015; Kaviraj et al. 2019), X-ray observations (e.g., Schramm et al. 2013; Baldassare et al. 2015; Mezcua et al. 2016; Chen et al. 2017; Kawamuro et al. 2019), as well as through studies of the AGN flux variability based on the properties that lower luminosity AGN have stronger variability amplitude (Morokuma et al. 2016; Baldassare et al. 2018, 2020; Elmer et al. 2020; Kimura et al. 2020). However, radio surveys have rarely been used for searching massive BHs in low-mass galaxies.

Recently, Reines et al. (2020) employed the radio-survey approach to search for massive BHs by using VLA/FIRST and conducting VLA high spatial resolution follow-up observations of local dwarf galaxies at $z<0.1$ and found more than 10 candidates. One remarkable result was that most radio cores were not located at the center of the host, but off-nucleus, a possible signature of a previous merger. If the detection of an off-nucleus core is indeed the consequence of a galaxy merger, it indicates that the orbital decay of a black hole through dynamical friction is inefficient in this $\mathrm{BH}$ mass range, resulting in a population of $\mathrm{BHs}$ that fail to reach the galactic center within a Hubble time since major mergers in dwarf galaxies becomes rare at $z<3$ (Fitts et al. 2018). Currently there are many theoretical implications regarding wandering BHs (Comerford \& Greene 2014; Blecha et al. 2016; Tremmel et al. 2018; De Rosa et al. 2019). High spatial resolution radio imaging can pin down the locations of such wandering BHs, and large-scale statistical studies may provide insights into the frequency and environment of wandering BHs through the combination of LSST and VLA/FIRST or the ongoing VLASS (Lacy et al. 2020), which will achieve a sensitivity down to $0.1 \mathrm{mJy}$ at $2-4 \mathrm{GHz}$, giving an order of magnitude deeper radio observations than VLA/FIRST.

\subsection{Do Blazars Contaminate ERGs?}

It could be argued that blazars might contaminate the sample of ERGs, and that therefore the optical emission is dominated by the jet component. Based on the known blazar sequence in the radio and optical bands (Fossati et al. 1998; Donato et al. 2001; Inoue \& Totani 2009; Ghisellini et al. 2017), the observed radio loudness of blazars is in the range of $\log \mathcal{R}_{\mathrm{obs}} \sim 1.5-3$. This range is obtained by using the luminosity range of $L_{1.4 \mathrm{GHz}}=10^{41-43}$ $\mathrm{erg} \mathrm{s}^{-1}$ and shifting redshift to the range of $z \sim 0-5$. Therefore, blazars cannot reproduce extreme radio loudness reaching $\log \mathcal{R}_{\text {rest }}>4$. Blazar contamination is unlikely also from the point of the observed optical magnitude. Considering that all ERGs are VLA/FIRST selected with a flux limit of $>1 \mathrm{mJy}$, the expected observed magnitude of blazars based on the blazar sequence is high with a peak around $i_{\mathrm{AB}}=21$, and all cases are at $i_{\mathrm{AB}}<23.5$ even when changing the luminosity range of the blazar SEDs and also shifting the redshift range to $z=0-5$. Based on these arguments, the contamination of blazars on ERGs is unlikely. 


\section{Conclusion}

The long-missing optical counterparts of the radio-bright $\left(f_{\nu}>1 \mathrm{mJy}\right.$ at $\left.1.4 \mathrm{GHz}\right)$ VLA/FIRST population have been constructed by Yamashita et al. (2018) by utilizing the wide $\left(>100 \mathrm{deg}^{2}\right)$ and deep $\left(i_{\mathrm{AB}}<26\right)$ Subaru/HSC SSP survey (named WERGS). The WERGS catalog contains a unique radio galaxy population with $\log \mathcal{R}_{\text {rest }}>4$ or extremely radioloud galaxies (ERGs). The number density of ERGs indicates that they are a very rare population $\left(\sim 1\right.$ source $\left.\mathrm{deg}^{-2}\right)$ and therefore it is not surprising that ERGs were missed in previous optical counterpart surveys of the radio sources. Combining multi-wavelength data in optical, IR, and radio bands (Toba et al. 2019), we have compiled a sample of 39 ERGs and 987 NRGs with radio-loudness is $1<\log \mathcal{R}_{\text {rest }}<4$.

We have investigated the properties of these unique ERGs and found the following key results:

1. Although the estimated SFR might have large uncertainties with $\sim 0.5$ dex, all ERGs are likely in the star-forming or starburst phase reaching a specific star formation rate of sSFR $\sim 10^{-8} \mathrm{yr}^{-1}$, suggesting that ERGs might be in a rapid stellar-mass assembly phase with mass-doubling times of $\sim 100 \mathrm{Myr}$. Besides, their stellar mass is relatively small, including the low-mass galaxies with $M_{\star}<10^{10} M_{\odot}$.

2. IR detected ERGs are in a rapid BH accretion phase with high specific black hole accretion rate (sBHAR) with the expected Eddington ratio $\left\langle\log \lambda_{\text {Edd }}\right\rangle \approx-0.4$ and some ERGs may be experiencing a super-Eddington phase.

3. Sources with higher $\mathcal{R}_{\text {rest }}$ tend to have both higher sBHAR (and thus higher $\lambda_{\text {Edd }}$ ) and higher intrinsic radio loudness $\mathcal{R}_{\text {int }}$. This paints a different picture of radio galaxies compared to conventionally known local radio galaxies with low $\lambda_{\text {Edd. }}$. ERGs represent a population of unique radio galaxies characterized by both high $\lambda_{\mathrm{Edd}}$ and high radio power.

4. The jet efficiencies of ERGs are typically $\eta_{\text {jet }} \sim 0.1$, which is similar to local radio AGNs residing in massive galaxies, whereas it is an order of magnitude higher than the values estimated for SDSS radio-loud quasars. This indicates that, although the accretion disk is reaching super-Eddington accretion, it is in a radiatively inefficient phase (possibly in a slim disk configuration) due to rapid mass accretion onto the central $\mathrm{BH}$.

We thank the anonymous referee for helpful suggestions that strengthened the paper. We thank Yoshiyuki Inoue for providing us the blazar sequence SED templates. This work is supported by Program for Establishing a Consortium for the Development of Human Resources in Science and Technology, Japan Science and Technology Agency (JST) and is partially supported by Japan Society for the Promotion of Science (JSPS) KAKENHI (18K13584 and 20H01939; K. Ichikawa, 18J01050 and 19K14759; Y. Toba, 16H03958, 17H01114, 19H00697; T. Nagao, 17J09016: T. Kawamuro, 19K03862: A.Y. Wagner). M. Charisi acknowledges support from the National Science Foundation (NSF) NANOGrav Physics Frontier Center, award number 1430284.

The Hyper Suprime-Cam (HSC) collaboration includes the astronomical communities of Japan and Taiwan, and Princeton University. The HSC instrumentation and software were developed by the National Astronomical Observatory of Japan
(NAOJ), the Kavli Institute for the Physics and Mathematics of the Universe (Kavli IPMU), the University of Tokyo, the HighEnergy Accelerator Research Organization (KEK), the Academia Sinica Institute for Astronomy and Astrophysics in Taiwan (ASIAA), and Princeton University. Funding was contributed by the FIRST program from the Japanese Cabinet Office, the Ministry of Education, Culture, Sports, Science and Technology (MEXT), the Japan Society for the Promotion of Science (JSPS), Japan Science and Technology Agency (JST), the Toray Science Foundation, NAOJ, Kavli IPMU, KEK, ASIAA, and Princeton University.

This paper makes use of software developed for the Large Synoptic Survey Telescope. We thank the LSST Project for making their code available as free software at http://dm. lsst.org.

This paper is based on data collected at the Subaru Telescope and retrieved from the HSC data archive system, which is operated by the Subaru Telescope and Astronomy Data Center (ADC) at NAOJ. Data analysis was in part carried out with the cooperation of the Center for Computational Astrophysics (CfCA), NAOJ.

The Pan-STARRS1 Surveys (PS1) and the PS1 public science archive have been made possible through contributions by the Institute for Astronomy, the University of Hawaii, the Pan-STARRS Project Office, the Max Planck Society and its participating institutes, the Max Planck Institute for Astronomy, Heidelberg, and the Max Planck Institute for Extraterrestrial Physics, Garching, The Johns Hopkins University, Durham University, the University of Edinburgh, the Queens University Belfast, the Harvard-Smithsonian Center for Astrophysics, the Las Cumbres Observatory Global Telescope Network Incorporated, the National Central University of Taiwan, the Space Telescope Science Institute, the National Aeronautics and Space Administration under grant No. NNX08AR22G issued through the Planetary Science Division of the NASA Science Mission Directorate, the National Science Foundation grant No. AST-1238877, the University of Maryland, Eotvos Lorand University (ELTE), the Los Alamos National Laboratory, and the Gordon and Betty Moore Foundation.

Facilities: VLA, Herschel, WISE, Subaru/HSC.

Software: astropy (Astropy Collaboration et al. 2013), Matplotlib (Hunter 2007), Pandas (McKinney 2010).

\section{Appendix A \\ How Much Does Wrong Photo-z Affect Our Results?}

Our results on ERGs rely on photo-z estimates, which affect physical values calculated using luminosity distance, e.g., stellar mass and luminosities. Unfortunately, because most of our sources are optically faint, there are no spec- $z$ confirmed ERGs in our sample. A concern is how erroneous photo- $z$ estimates may affect our results.

If our photo- $z$ values are severely underestimated, it is clear that the absolute physical values such as stellar mass, with the dependence of luminosity distance of $\mathrm{D}_{L}^{2}$, have been underestimated, and therefore the results on low-mass $\mathrm{BH}$ mass and low-mass galaxy arguments discussed in Section 4.4 are no longer valid. One situation in which this might happen is that our photo- $z$ estimation at $z \sim 0.3-0.5$ is not tracing the Balmer break at $\sim 4000 \AA$, but actually the Lyman break at $z \sim 3$ (e.g., Tanaka et al. 2018). 

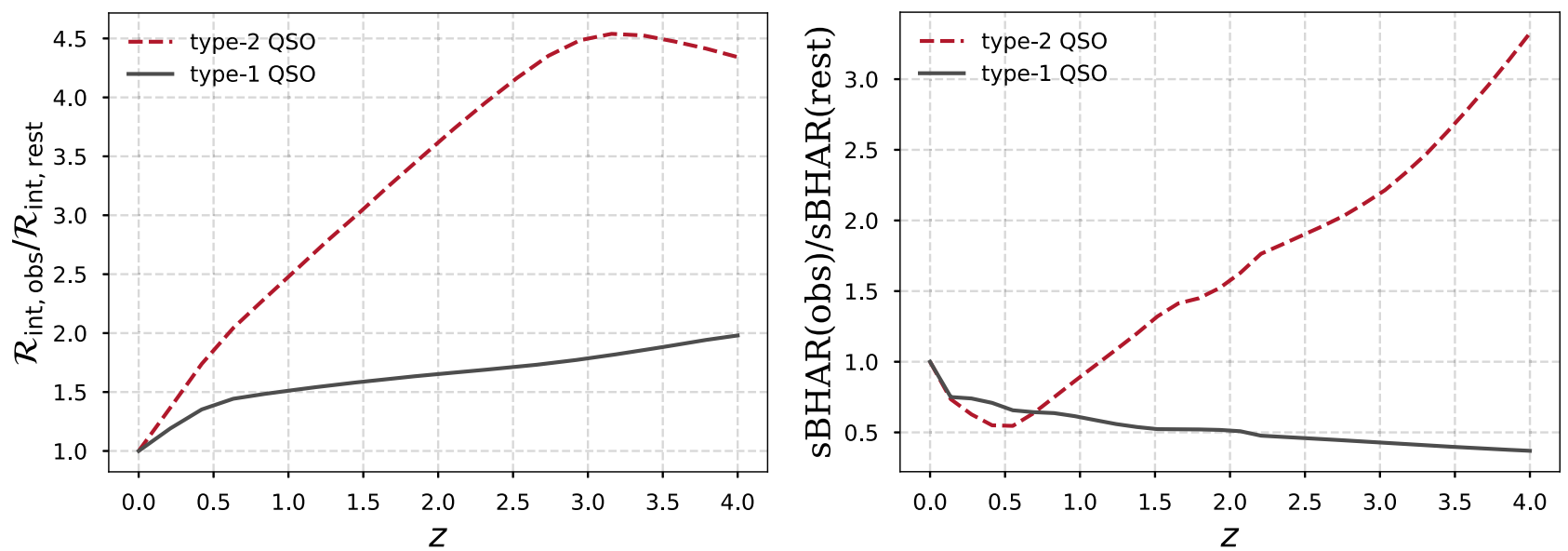

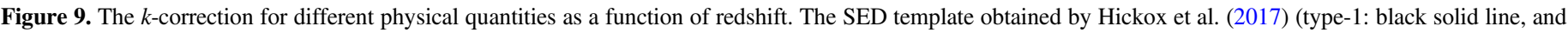

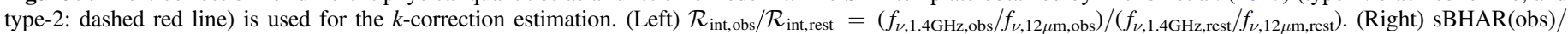
$\operatorname{sBHAR}($ rest $)=\left(f_{\nu, 12 \mu \mathrm{m}, \mathrm{obs}} / f_{\nu, i \text { band, obs }}\right) /\left(f_{\nu, 12 \mu \mathrm{m}, \text { rest }} / f_{\nu, i \text { band, rest }}\right)$.

However, physical quantities obtained from the ratios of two physical quantities have weaker redshift dependences. For both $\mathcal{R}_{\text {int }}$ and sBHAR, the luminosity distance dependence is canceled out. The remaining $z$-dependent factor is the $k$-correction between the observed flux and the rest frame flux. The physical quantities $L_{\text {jet }}, L_{\mathrm{AGN}, \mathrm{IR}}$ (and therefore $L_{\mathrm{AGN}, \mathrm{bol}}$ ), and $M_{\star}$, can be mainly traced by one-band flux (densities) of FIRST $1.4 \mathrm{GHz}$ tracing radio Synchrotron emission $\left(f_{\nu, 1.4 \mathrm{GHz}}\right)$, WISE MIR band(s) tracing the peak of AGN dust emission $\left(f_{\nu, 12 \mu \mathrm{m}}\right)$, and the $i$ band tracing stellar emission $\left(f_{\nu, i \text { band }}\right)$. Therefore, each physical quantity can be written as $\mathcal{R}_{\text {int }}=L_{\text {jet }} / L_{\mathrm{AGN}, \text { bol }} \propto f_{\nu, 1.4 \mathrm{GHz}} / f_{\nu, 12 \mu \mathrm{m}}$ and $\mathrm{sBHAR}=L_{\mathrm{AGN}, \mathrm{bol}} / M_{\star} \propto f_{\nu, 12 \mu \mathrm{m}} / f_{\nu, i \text { band. }}$. We checked and confirmed the proportionality of these ratios in our sample.

We then investigated the $k$-correction by seeing how much these observed values change as a function of redshift. In the radio band, we assumed the same power-law $f_{\nu} \propto \nu^{\alpha}$ with $\alpha=-0.7$. In the optical and IR band, we used the quasar SED templates obtained by Hickox et al. (2017), which contain two templates of type- 1 and type- 2 quasars, whose optical band is dominated by accretion disk and stellar component, respectively. Since the SED extends only down to $2000 \AA$, we extrapolated the SED with $\alpha=-0.5$ for type-1 QSOs and $\alpha=-2.4$ for type-2 QSOs in order to smoothly connect the SED template (e.g., see also Richards et al. 2006; Polletta et al. 2007, for other SED templates). Given their high radio loudness and as we discussed in Section 3, the SED of ERGs is likely similar to that of type-2 QSOs, whose optical emission is dominated by the stellar component.

Figure 9 shows the $k$-correction of each physical value as a function of redshift. The $k$-correction changes by a factor of up to 4.5 for $\mathcal{R}_{\text {int }}$ and factor of 3 for sBHAR even when the targets are shifted at $z \sim 4$. This suggests that an erroneous photo- $z$ does not strongly affect our overall results for $\mathcal{R}_{\text {int }}$ and sBHAR.

\section{Appendix B Redshift Dependence of Our Results}

The physical properties between ERGs and NRGs are explored in Section 3 and discussed in Section 4. As shown in
Figure 2, NRGs distribute smoothly in a wide redshift range at $0.3<z<1.6$, while ERGs are slightly clustered at $z>0.8$. Thus, one might wonder whether the difference in physical parameters between ERGs and NRGs is caused by the redshift difference. We here split the sample into the three redshift bins $(z=0.3-0.8,0.8-1.2$, and 1.2-1.6) for mitigating such redshift dependence and then compare the key parameters of the two populations.

The top panels of Figure 10 show the relations between SFR and $M_{\star}$, which are similar to ones with Figure 3 . Most of the NRGs reside below the MS in the $0.3<z<0.8$, then the fraction of sources on and above the MS increases as a function of redshift. However, ERGs always locate the lower $M_{\star}$ range in each redshift bin and most of them are above the MS (except several sources on and below the MS). Considering that the stellar mass is estimated from the photometries mainly in the optical or near-IR bands, the deeper Subaru/HSC photometries enable us to find this smaller stellar-mass population as shown in Figure 4.

The middle panels of Figure 10 show the relations between sBHAR and $M_{\star}$, which are the redshift-divided version of Figure 5. At $0.3<z<0.8$, most of the NRGs are located at low sBHAR and high $M_{\star}$ cluster region where sBHAR/erg $\mathrm{s}^{-1} M_{\odot}^{-1} \sim 10^{33.5}$ and $M_{\star} \sim 10^{11.5} M_{\odot}$, and then they move to higher sBHAR sequence with redshift. However, ERGs always locate at the top edge of the sequence, keeping high $\mathrm{SBHAR}$ values and sometimes reaching the sBHAR equivalent to $\lambda_{\text {Edd }} \sim 1$.

Finally, the bottom panels of Figure 10 show the relation between $\mathcal{R}_{\text {int }}$ and sBHAR, which are the redshift-divided ones of Figure 7. Most of the NRGs are located at the top left region at $0.3<z<0.8$, and then the population moves to higher sBHAR and smaller $\mathcal{R}_{\text {int }}$ sequence, moving to bottom-center or bottom-right of the panel. This is a consistent view of local radio galaxies at lower- $z$ and radio quasar population at higher$z$ as discussed in Section 4.2. However, ERGs reside in the top right part of the plane, and does not show a redshift evolution, because of its extreme selection cut of $\log \mathcal{R}_{\text {rest }}>4$, limiting the sample only in the top right area of the panel. 

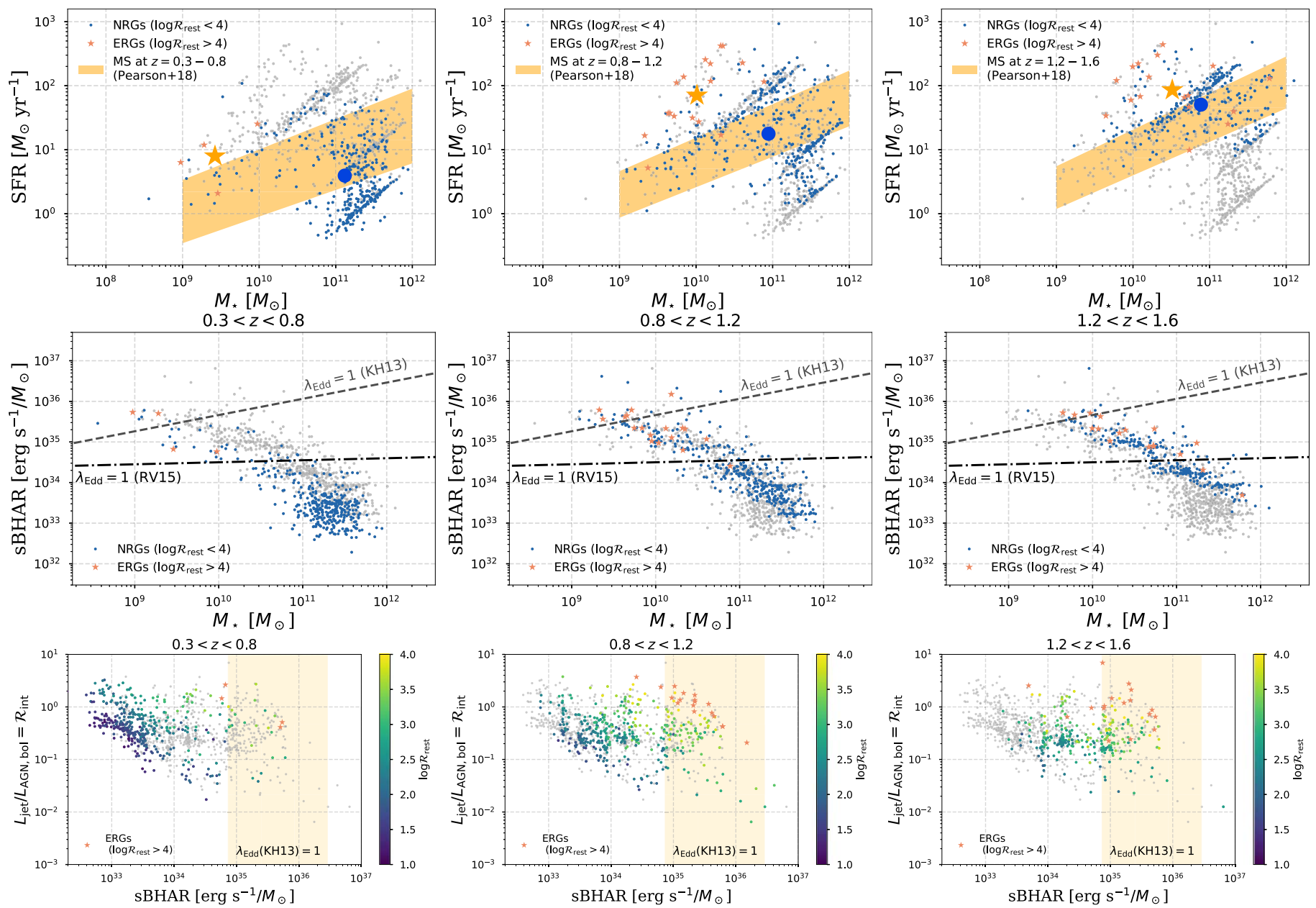

Figure 10. The same relations but with a redshift-divided sample of $0.3<z<0.8$ (Left), $0.8<z<1.2$ (Center), and $1.2<z<1.6$ (Right) overlapped with the total sample $(0.3<z<1.6)$ in gray. (Top) The relation between SFR and stellar mass $\left(M_{\star}\right)$ and the symbols are the same as in Figure 3. (Middle) The relation between sBHAR and $M_{\star}$, same with Figure 5. (Bottom) The relationship between $L_{\text {jet }} / L_{\mathrm{AGN}, \text { bol }}=\mathcal{R}_{\text {int }}$ and sBHAR, same with Figure 7.

\section{ORCID iDs}

Kohei Ichikawa (1) https://orcid.org/0000-0002-4377-903X

Takuji Yamashita (i) https://orcid.org/0000-0002-4999-9965

Yoshiki Toba (10 https://orcid.org/0000-0002-3531-7863

Tohru Nagao (1) https://orcid.org/0000-0002-7402-5441

Kohei Inayoshi (1) https://orcid.org/0000-0001-9840-4959

Maria Charisi (i) https://orcid.org/0000-0003-3579-2522

Alexander Y. Wagner (i) https://orcid.org/0000-0002-

5104-6434

Masayuki Akiyama (ํ) https://orcid.org/0000-0002-2651-1701

Xiaoyang Chen (1) https://orcid.org/0000-0003-2682-473X

Masaru Kajisawa ำ https://orcid.org/0000-0002-1732-6387

Taiki Kawamuro (1) https://orcid.org/0000-0002-6808-2052

Chien-Hsiu Lee (10) https://orcid.org/0000-0003-1700-5740

Malte Schramm (1) https://orcid.org/0000-0001-7825-0075

Hyewon Suh (1) https://orcid.org/0000-0002-2536-1633

Masayuki Tanaka (i] https://orcid.org/0000-0002-5011-5178

Yoshihiro Ueda (1) https://orcid.org/0000-0001-7821-6715

\section{References}

Abramowicz, M. A., Czerny, B., Lasota, J. P., \& Szuszkiewicz, E. 1988, ApJ, 332,646

Aihara, H., Arimoto, N., Armstrong, R., et al. 2018a, PASJ, 70, S4

Aihara, H., Armstrong, R., Bickerton, S., et al. 2018b, PASJ, 70, S8
Aird, J., Coil, A. L., \& Georgakakis, A. 2019, MNRAS, 484, 4360

Aird, J., Coil, A. L., Moustakas, J., et al. 2012, ApJ, 746, 90

Alam, S., Albareti, F. D., Allende Prieto, C., et al. 2015, ApJS, 219, 12

Asmus, D., Gandhi, P., Hönig, S. F., Smette, A., \& Duschl, W. J. 2015, MNRAS, 454, 766

Astropy Collaboration, Robitaille, T. P., Tollerud, E. J., et al. 2013, A\&A, 558, A33

Baldassare, V. F., Geha, M., \& Greene, J. 2018, ApJ, 868, 152

Baldassare, V. F., Geha, M., \& Greene, J. 2020, ApJ, 896, 10

Baldassare, V. F., Reines, A. E., Gallo, E., \& Greene, J. E. 2015, ApJL, 809, L14

Bañados, E., Venemans, B. P., Mazzucchelli, C., et al. 2018, Natur, 553, 473

Barth, A. J., Greene, J. E., \& Ho, L. C. 2008, AJ, 136, 1179

Becker, R. H., White, R. L., \& Helfand, D. J. 1995, ApJ, 450, 559

Best, P. N., \& Heckman, T. M. 2012, MNRAS, 421, 1569

Best, P. N., Kauffmann, G., Heckman, T. M., \& Ivezić, Ž. 2005, MNRAS, 362,9

Bîrzan, L., McNamara, B. R., Nulsen, P. E. J., Carilli, C. L., \& Wise, M. W. 2008, ApJ, 686, 859

Blandford, R., Meier, D., \& Readhead, A. 2019, ARA\&A, 57, 467

Blandford, R. D., \& Znajek, R. L. 1977, MNRAS, 179, 433

Blecha, L., Sijacki, D., Kelley, L. Z., et al. 2016, MNRAS, 456, 961

Boquien, M., Burgarella, D., Roehlly, Y., et al. 2019, A\&A, 622, A103

Bosch, J., Armstrong, R., Bickerton, S., et al. 2018, PASJ, 70, S5

Bower, R. G., Schaye, J., Frenk, C. S., et al. 2017, MNRAS, 465, 32

Brinchmann, J., Charlot, S., White, S. D. M., et al. 2004, MNRAS, 351, 1151

Cavagnolo, K. W., McNamara, B. R., Nulsen, P. E. J., et al. 2010, ApJ, 720, 1066

Chen, C. T. J., Brandt, W. N., Reines, A. E., et al. 2017, ApJ, 837, 48

Ciesla, L., Elbaz, D., \& Fensch, J. 2017, A\&A, 608, A41 
Comerford, J. M., \& Greene, J. E. 2014, ApJ, 789, 112

Condon, J. J. 1992, ARA\&A, 30, 575

Condon, J. J., Kellermann, K. I., Kimball, A. E., Ivezić, Ž., \& Perley, R. A. 2013, ApJ, 768, 37

Costa, T., Rosdahl, J., Sijacki, D., \& Haehnelt, M. G. 2018, MNRAS, 479, 2079

De Rosa, A., Vignali, C., Bogdanović, T., et al. 2019, NewAR, 86, 101525

Decarli, R., Walter, F., Venemans, B. P., et al. 2018, ApJ, 854, 97

Del Moro, A., Alexander, D. M., Mullaney, J. R., et al. 2013, A\&A, 549, A59

Delvecchio, I., Gruppioni, C., Pozzi, F., et al. 2014, MNRAS, 439, 2736

Delvecchio, I., Smolčić, V., Zamorani, G., et al. 2018, MNRAS, 481, 4971

Dickey, C., Geha, M., Wetzel, A., \& El-Badry, K. 2019, ApJ, 884, 180

Ding, X., Silverman, J., Treu, T., et al. 2020, ApJ, 888, 37

Donato, D., Ghisellini, G., Tagliaferri, G., \& Fossati, G. 2001, A\&A, 375, 739

Drinkwater, M. J., Jurek, R. J., Blake, C., et al. 2010, MNRAS, 401, 1429

Driver, S. P., Hill, D. T., Kelvin, L. S., et al. 2011, MNRAS, 413, 971

Elbaz, D., Dickinson, M., Hwang, H. S., et al. 2011, A\&A, 533, A119

Elmer, E., Almaini, O., Merrifield, M., et al. 2020, MNRAS, 493, 3026

Ferrarese, L., \& Merritt, D. 2000, ApJL, 539, L9

Fitts, A., Boylan-Kolchin, M., Bullock, J. S., et al. 2018, MNRAS, 479, 319

Fossati, G., Maraschi, L., Celotti, A., Comastri, A., \& Ghisellini, G. 1998, MNRAS, 299, 433

Furusawa, H., Koike, M., Takata, T., et al. 2018, PASJ, 70, S3

Gaibler, V., Khochfar, S., Krause, M., \& Silk, J. 2012, MNRAS, 425, 438

Gandhi, P., Horst, H., Smette, A., et al. 2009, A\&A, 502, 457

Gebhardt, K., Bender, R., Bower, G., et al. 2000, ApJL, 539, L13

Ghisellini, G., Righi, C., Costamante, L., \& Tavecchio, F. 2017, MNRAS, 469,255

Greene, J. E., \& Ho, L. C. 2004, ApJ, 610, 722

Greene, J. E., \& Ho, L. C. 2007, ApJ, 667, 131

Greene, J. E., Strader, J., \& Ho, L. C. 2020, ARA\&A, 58, 257

Habouzit, M., Volonteri, M., \& Dubois, Y. 2017, MNRAS, 468, 3935

Haiman, Z., Brandt, W. N., Vikhlinin, A., et al. 2019, BAAS, 51, 557

Häring, N., \& Rix, H.-W. 2004, ApJL, 604, L89

Helfand, D. J., White, R. L., \& Becker, R. H. 2015, ApJ, 801, 26

Helou, G., Soifer, B. T., \& Rowan-Robinson, M. 1985, ApJL, 298, L7

Hickox, R. C., Jones, C., Forman, W. R., et al. 2009, ApJ, 696, 891

Hickox, R. C., Myers, A. D., Greene, J. E., et al. 2017, ApJ, 849, 53

Ho, L. C. 2002, ApJ, 564, 120

Ho, L. C. 2008, ARA\&A, 46, 475

Holt, J., Tadhunter, C. N., \& Morganti, R. 2008, MNRAS, 387, 639

Huang, S., Leauthaud, A., Murata, R., et al. 2018, PASJ, 70, S6

Hunter, J. D. 2007, CSE, 9, 90

Ichikawa, K., \& Inayoshi, K. 2017, ApJL, 840, L9

Ichikawa, K., Ricci, C., Ueda, Y., et al. 2017, ApJ, 835, 74

Ichikawa, K., Ricci, C., Ueda, Y., et al. 2019, ApJ, 870, 31

Ichikawa, K., Ueda, Y., Terashima, Y., et al. 2012, ApJ, 754, 45

Inayoshi, K., \& Haiman, Z. 2016, ApJ, 828, 110

Inayoshi, K., Haiman, Z., \& Ostriker, J. P. 2016, MNRAS, 459, 3738

Inayoshi, K., Ichikawa, K., \& Haiman, Z. 2018, ApJL, 863, L36

Inayoshi, K., Visbal, E., \& Haiman, Z. 2020, ARA\&A, 58, 27

Inoue, Y., Doi, A., Tanaka, Y. T., Sikora, M., \& Madejski, G. M. 2017, ApJ, 840,46

Inoue, Y., \& Totani, T. 2009, ApJ, 702, 523

Intema, H. T., Jagannathan, P., Mooley, K. P., \& Frail, D. A. 2017, A\&A, 598, A78

Ivison, R. J., Magnelli, B., Ibar, E., et al. 2010, A\&A, 518, L31 Ivezić, Ž., Kahn, S. M., Tyson, J. A., et al. 2019, ApJ, 873, 111 Ivezić, Ž., Menou, K., Knapp, G. R., et al. 2002, AJ, 124, 2364 Kaviraj, S., Martin, G., \& Silk, J. 2019, MNRAS, 489, L12

Kawamuro, T., Ueda, Y., Ichikawa, K., et al. 2019, ApJ, 881, 48

Kawanomoto, S., Uraguchi, F., Komiyama, Y., et al. 2018, PASJ, 70, 66

Kimball, A. E., Kellermann, K. I., Condon, J. J., Ivezić, Ž., \& Perley, R. A. 2011, ApJL, 739, L29

Kimura, Y., Yamada, T., Kokubo, M., et al. 2020, ApJ, 894, 24

Komiyama, Y., Obuchi, Y., Nakaya, H., et al. 2018, PASJ, 70, S2

Kormendy, J., \& Ho, L. C. 2013, ARA\&A, 51, 511

Lacy, M., Baum, S. A., Chandler, C. J., et al. 2020, PASP, 132, 035001

Läsker, R., Greene, J. E., Seth, A., et al. 2016, ApJ, 825, 3

Liske, J., Baldry, I. K., Driver, S. P., et al. 2015, MNRAS, 452, 2087

Magorrian, J., Tremaine, S., Richstone, D., et al. 1998, AJ, 115, 2285

Maiolino, R., Russell, H. R., Fabian, A. C., et al. 2017, Natur, 544, 202

Marconi, A., \& Hunt, L. K. 2003, ApJL, 589, L21
McConnell, N. J., \& Ma, C.-P. 2013, ApJ, 764, 184

McKinney, J. C., Tchekhovskoy, A., Sadowski, A., \& Narayan, R. 2014, MNRAS, 441, 3177

McKinney, W. 2010, in Proc. 9th Python in Science Conf., ed. S. van der Walt \& J. Millman (Austin, TX: SciPy), 51

McLure, R. J., \& Dunlop, J. S. 2004, MNRAS, 352, 1390

McNamara, B. R., \& Nulsen, P. E. J. 2007, ARA\&A, 45, 117

McQuinn, K. B. W., Skillman, E. D., Cannon, J. M., et al. 2010, ApJ, 724, 49

Merloni, A., Bongiorno, A., Bolzonella, M., et al. 2010, ApJ, 708, 137

Merloni, A., \& Heinz, S. 2007, MNRAS, 381, 589

Mezcua, M., Civano, F., Fabbiano, G., Miyaji, T., \& Marchesi, S. 2016, ApJ, 817,20

Mezcua, M., Suh, H., \& Civano, F. 2019, MNRAS, 488, 685

Miyazaki, S., Komiyama, Y., Kawanomoto, S., et al. 2018, PASJ, 70, S1

Morganti, R., Tadhunter, C. N., \& Oosterloo, T. a. 2005, A\&A, 444, L9

Morokuma, T., Tominaga, N., Tanaka, M., et al. 2016, PASJ, 68, 40

Mortlock, D. J., Warren, S. J., Venemans, B. P., et al. 2011, Natur, 474, 616

Mukherjee, D., Bicknell, G. V., Wagner, A. Y., Sutherland, R. S., \& Silk, J. 2018, Mon. Not. R. Astron. Soc, 479, 5544

Mullaney, J. R., Daddi, E., Béthermin, M., et al. 2012, ApJL, 753, L30

Nemmen, R. S., \& Tchekhovskoy, A. 2015, MNRAS, 449, 316

Nesvadba, N. P. H., De Breuck, C., Lehnert, M. D., Best, P. N., \& Collet, C. 2017, A\&AS, 599, A123

Noeske, K. G., Weiner, B. J., Faber, S. M., et al. 2007, ApJL, 660, L43

Nyland, K., Davis, T. A., Nguyen, D. D., et al. 2017, ApJ, 845, 50

Ohsuga, K., Mineshige, S., Mori, M., \& Kato, Y. 2009, PASJ, 61, L7

Ohsuga, K., Mori, M., Nakamoto, T., \& Mineshige, S. 2005, ApJ, 628, 368

Onoue, M., Kashikawa, N., Matsuoka, Y., et al. 2019, ApJ, 880, 77

O'Sullivan, E., Giacintucci, S., David, L. P., et al. 2011, ApJ, 735, 11

Padovani, P. 2016, A\&ARv, 24, 13

Panessa, F., Barcons, X., Bassani, L., et al. 2007, A\&A, 467, 519

Pearson, W. J., Wang, L., Hurley, P. D., et al. 2018, A\&A, 615, A146

Penny, S. J., Masters, K. L., Smethurst, R., et al. 2018, MNRAS, 476, 979

Polletta, M., Tajer, M., Maraschi, L., et al. 2007, ApJ, 663, 81

Rafferty, D. A., McNamara, B. R., Nulsen, P. E. J., \& Wise, M. W. 2006, ApJ, 652,216

Reines, A. E., Condon, J. J., Darling, J., \& Greene, J. E. 2020, ApJ, 888, 36

Reines, A. E., Greene, J. E., \& Geha, M. 2013, ApJ, 775, 116

Reines, A. E., \& Volonteri, M. 2015, ApJ, 813, 82

Richards, G. T., Lacy, M., Storrie-Lombardi, L. J., et al. 2006, ApJS, 166, 470

Sadowski, A., \& Narayan, R. 2015, MNRAS, 453, 3213

Sadowski, A., Narayan, R., McKinney, J. C., \& Tchekhovskoy, A. 2014, MNRAS, 439, 503

Sani, E., Marconi, A., Hunt, L. K., \& Risaliti, G. 2011, MNRAS, 413, 1479

Sartori, L. F., Schawinski, K., Treister, E., et al. 2015, MNRAS, 454, 3722

Satyapal, S., Secrest, N. J., McAlpine, W., et al. 2014, ApJ, 784, 113

Schinnerer, E., Smolčić, V., Carilli, C. L., et al. 2007, ApJS, 172, 46

Schramm, M., Silverman, J. D., Greene, J. E., et al. 2013, ApJ, 773, 150

Schreiber, C., Pannella, M., Elbaz, D., et al. 2015, A\&A, 575, A74

Secrest, N. J., Satyapal, S., Gliozzi, M., et al. 2015, ApJ, 798, 38

Seymour, N., Stern, D., De Breuck, C., et al. 2007, ApJS, 171, 353

Shabala, S. S., \& Godfrey, L. E. H. 2013, ApJ, 769, 129

Sikora, M., Stawarz, Ł., \& Lasota, J.-P. 2007, ApJ, 658, 815

Sikora, M., Stasińska, G., Kozieł-Wierzbowska, D., Madejski, G. M., \& Asari, N. V. 2013, ApJ, 765, 62

Silk, J. 2017, ApJL, 839, L13

Smolčić, V., Delvecchio, I., Zamorani, G., et al. 2017b, A\&A, 602, A2

Smolčić, V., Novak, M., Bondi, M., et al. 2017a, A\&A, 602, A1

Soltan, A. 1982, MNRAS, 200, 115

Tadhunter, C. 2016, A\&ARv, 24, 10

Takada, M., Ellis, R. S., Chiba, M., et al. 2014, PASJ, 66, R1

Takeo, E., Inayoshi, K., \& Mineshige, S. 2020, MNRAS, 497, 302

Tanaka, M. 2015, ApJ, 801, 20

Tanaka, M., Coupon, J., Hsieh, B.-C., et al. 2018, PASJ, 70, S9

Tchekhovskoy, A., Narayan, R., \& McKinney, J. C. 2011, MNRAS, 418, L79

Terashima, Y., \& Wilson, A. S. 2003, ApJ, 583, 145

Toba, Y., Yamashita, T., Nagao, T., et al. 2019, ApJS, 243, 15

Trakhtenbrot, B., Urry, C. M., Civano, F., et al. 2015, Sci, 349, 168

Tremaine, S., Gebhardt, K., Bender, R., et al. 2002, ApJ, 574, 740

Tremmel, M., Governato, F., Volonteri, M., Pontzen, A., \& Quinn, T. R. 2018, ApJL, 857, L22

van Velzen, S., \& Falcke, H. 2013, A\&A, 557, L7 
Wagner, A. Y., \& Bicknell, G. V. 2011, ApJ, 728, 29

Wagner, A. Y., Bicknell, G. V., Umemura, M., Sutherland, R. S., \& Silk, J. 2016, AN, 337, 167

Wang, R., Wagg, J., Carilli, C. L., et al. 2013, ApJ, 773, 44

Wang, R., Wu, X.-B., Neri, R., et al. 2016, ApJ, 830, 53

Whitaker, K. E., van Dokkum, P. G., Brammer, G., \& Franx, M. 2012, ApJL, 754, L29

White, R. L., Becker, R. H., Helfand, D. J., \& Gregg, M. D. 1997, ApJ, 475, 479
Willott, C. J., Rawlings, S., Blundell, K. M., \& Lacy, M. 1999, MNRAS, 309, 1017

Woo, J.-H., Treu, T., Malkan, M. A., \& Bland ford, R. D. 2008, ApJ, 681, 925

Wu, X.-B., Wang, F., Fan, X., et al. 2015, Natur, 518, 512

Xiao, T., Barth, A. J., Greene, J. E., et al. 2011, ApJ, 739, 28

Yamashita, T., Nagao, T., Akiyama, M., et al. 2018, ApJ, 866, 140

Yamashita, T., Nagao, T., \& Ikeda, H. 2020, ApJ, 160, 60 